\title{
Anthoc Based Trust Behavioural Network (Anthoc- tbn) Assist to Detect Blackhole Attack by Discover Secure Route
}

\author{
Rajkumar M ( $\sim$ rajkumarphd4@gmail.com ) \\ Sri Krishna College of Technology \\ Karthika J \\ KPR Institute of Engineering and Technology
}

\section{Research Article}

Keywords: Mobile Ad hoc Network, Ant Colony Optimization, Routing Table, Extended Data Routing Information, Blackhole Attack

Posted Date: June 8th, 2021

DOI: https://doi.org/10.21203/rs.3.rs-474353/v1

License: (c) (i) This work is licensed under a Creative Commons Attribution 4.0 International License.

Read Full License 


\title{
ANTHOC BASED TRUST BEHAVIOURAL NETWORK (ANTHOC-TBN) ASSIST TO DETECT BLACKHOLE ATTACK BY DISCOVER SECURE ROUTE
}

\author{
${ }^{1}$ Dr. Rajkumar M, ${ }^{2}$ Dr. Karthika M \\ ${ }^{1}$ Assistant Professor, Department of Information Technology, Sri krishna College of Technology, Coimbatore, \\ Tamilnadu, India.E-mail : rajkumarphd4@gmail.com \\ ${ }^{2}$ Professor, Department of Computer Science and Engineering, KPR Institute of Engineering and Technology. \\ Coimbatore, Tamilnadu, India. E-mail : j.karthikaphd@gmail.com \\ Corresponding Author : Dr. M Rajkumar \\ Corresponding Author E-mail : rajkumarphd4@gmail.com
}

\begin{abstract}
MANET is a wireless topology mainly used to grouping several mobile nodes as network. This wireless network is highly vulnerable by several security threads. Blackhole attack, a severe thread that voluntarily disrupts the healthy nodes and turn it as malicious node. So, introducing security mechanism can forcefully work together to defeat the black hole attack. In this paper, a new approach of AntHoc based trust behavioural network (AntHoc-TBN) model is introduced. This model effectively discovers the route with trust based packet transmission take place. Here, the forward ant agent performs shortest distance computation to discover the route. So that, Dijikstra algorithm is employed to detect the shortest route of all other nodes from source. Then, trust procedure is applied to find that corresponding node is trustable or not. Based on trust threshold, the respective intermediate node is computed and stored the updated value in extended data routing information (EDRI) table. Once the condition is not satisfied, then updated value in EDRI table tends to be distrust node and the concerned node is eliminated from the routing table. Now, the trusted node becomes an active path after that the packet has to be sent. Likewise, the same process is repeated for backward ant agent to transfer the Ack message from destination to source. To simulate the process, the result reveals that the AntHoc-TBN model improves its efficiency by lowest delay, packet loss and routing overhead. And also, the technique gets highest value of packet delivery ratio. The performance measures of our proposed model achieves better outcome when compared to existing techniques such as AODV, AntNet and AntHoc Net.
\end{abstract}

Keywords: Mobile Ad hoc Network, Ant Colony Optimization, Routing Table, Extended Data Routing Information and Blackhole Attack.

\section{Introduction}

Mobile Ad hoc network (MANET) is an infrastructure less model formed by a collection of mobile nodes that configure the network via wireless link. The nodes in the MANET can move dynamically so that the nature of structural topology changes appropriately [1]. MANET is a self-configuring, quick and easy network setup designed by hop-hop communication and also it is specialized not only for data communication but also simply act as a router. Without the constraint of host movement, the cost effectiveness of MANET design is much lower. It uses mobile device as node that can move freely to arrive or depart the network at any regular interval. No required infrastructure and mobility features 
are the major factor that affects the security in MANET. The mechanism is that the MANET is harder to predict its threads because it doesn't have a centralized system. A few security threads arise in the MANET are denial-of-service (DoS), eavesdropping, blackhole, Wormhole, Man in the Middle attack etc.., [2]. A goal of attack is to disrupt the normal data and change them into malicious node which eventually misroute the packets. In order to solve these security threads, the researchers were used several mechanism such as interference based [3], node collaboration based [4], routing-based [5] and intrusion detection based [6] mechanism.

In this research, a multi-path routing protocol of AntHoc Net is used to maintain efficient routing discovery. Existing routing protocols can be categorized either as reactive or proactive [7, 8] but AntHoc Net contains both. In proactive routing protocols, it incessantly attempt to find entire routes within the network which in terms a flexible data forwarding procedure is possibly available. In addition, the latency is much reduced because the path is already known. Conversely, in reactive protocols, the technique used on-demand procedure to track the route based on global search. On-demand is extensively used routing protocol in most of the research works, because it consumes lower bandwidth than proactive routing protocols. Some of the on-demand routing protocols such as Dynamic Source Routing (DSR) [9] and Ad hoc On-Demand Distance Vector (AODV) [10] which is effectively demandable to discover the route. But, they discover unipath routing mechanism for data forwarding at each session. If any break arises in the active route, then routing undergoes significant latency during processing period. Hybrid routing protocol contains reactive and proactive that takes the both merits and conquer their demerits. AntHoc Net is a hybrid routing protocol that works under the principle of Ant Colony Optimization (ACO).It set up two paths namely initializing session for reactive forward ants and data forwarding session for proactive forward ants. It reacts to link failure either by warning preceding nodes or by local repair on the path.

The main objective of this research work is to implement an AntHoc based trust behavioural network (AntHoc-TBN) model in the MANET design. AntHoc Net routing discovery has been implemented with added such trust management in it. This trust model is embedded within the AntHoc Network, which primarily made a secure routing to detect malicious node. Trust model is effective and accurate mechanism that helps to identify the malicious node by creating the routing path as highly secured one. Based on routing procedure, trustworthy behaviour avoids the attacking nodes to create smooth data communication without loss of information. Finally, the designed structure has to be simulated using NS2 software and compute the effectiveness based on using measuring parameters such as routing overhead, delay, packet loss ratio and packet delivery ratio.

The research work is organized as follows, section 2 survey about various existing works relate to give solution for black hole attack, proposed framework of AntHoc based trust behavioural network (AntHoc-TBN) flow diagram and its description is detailed in section 3, Section 4 discuss the simulation result and performance measure and finally section 5 ends the work. 


\section{Related Works}

Black hole attack is the security thread that severely affects the unarranged mobile Ad hoc network (MANET).A proper safeguard measures and the solution are discussed in various existing papers. Some research works are listed as follows,

SinaShahabi et al. [11] presented an improved algorithm regarding security basis in MANET. This network topology used AODV routing protocol which allows path to both source and destination through two control packets namely Route Request (RREQ) and Route Response (RREP). This technique attempts to identify the destructive nodes within the network which is affected by black hole attack. Increase in destructive nodes may affect the delivery performance with heavy traffic. This complication may gradually increase the delay time. So, the difficulty is resolved by using IDSAODV protocol with certain ruling procedure. Based on the procedure, the request packet is passed between the nodes which send route response. Here, the received information from one node is considered as destructive node after then the route response break its connection and made it as quarantine with exposing danger alarm. With this alert mechanism, the unwanted destructive nodes are eradicated from the routing path. But the shortcoming arise in this technique is that the node contains flood of information.

Dost Muhammad Khan et al. [12] introduced a min-max ant system (MMAS) and reactive routing protocol suitably meant to prevent the black hole attack in MANET. In route discovery procedure, the ant colony optimization (ACO) used non-static components to discover their shortest path. Non-static is actually meant a pheromone chemical that makes a clear goal to find the optimum solution. The way of path selection between nodes is based on pheromone level. The next phase of route maintenance used is reactive routing protocol which constantly minimize the routing time. Invaluable for large-scale network, uncertainty in convergence time are few constraint available in this techniques.

Trust based DSR protocol is optimized with dynamic trust function which diminishes the cooperative black hole attack and is proposed by Ephantus Gichuki Mwangi et al. [13]. The basis of trust is to analyze the past experience of each node. Awarded a high trust level is promoted as more trusted node which in terms a successfully packet delivery takes place.

The optimized technique attain above $95 \%$ of packet delivery ratio and throughput as 95.6 Kbps. But this technique is significantly not effective to reduce the distributed black hole attack.

D.Mishra et al. [14] presented various clarifications to wipe out the black hole attack. Destination sequence number is detected first, so that the technique requires too many paths to reach the destination. This issue can creates huge delay and also packet bounce take place between the hubs. In order to give a favourable solution for this issue, I.S. Imad et al. [15] presented DPRAODV approach. It is actually a detection, prevention and reactive protocol which offer great tendency to identify malicious hubs. Referring the drawback, it occupies large memory space due to two distinct tables. 


\section{Proposed Framework}

In this section, a new trusted data communication modelling is proposed in the MANET. Collection of nodes is randomly arranged to form a network. Within the network, the ant agent performs route discovery process to check each node. AntHoc Net is the routing discovery protocol established to maintain promising routing facility for Mobile ad hoc network. The routing protocol uses two strategies to deliver the data from source to destination. They are,

- Find the shortest path between node

- Measure the trustable node

Both these processes can be effectively find shortest path between the nodes and also check trusted mechanism for the corresponding node. Therefore, the black node attack is being eliminated from the path to make a secure packet transmission. Under certain processing procedure, we designed a new trusted data communication scheme named as AntHoc based trust behavioural network (AntHoc-TBN) model.

\subsection{Ant Colony Optimization for routing}

It is a well known optimizer technique that inspired from ants behaviour. The main concept of this technique is to create communication between the nodes thereby resolve the routing problem. Practically, the biological ant drops some chemical substance (i.e., pheromone) on the routing path to communicate with each other. This substrate is the best tracking strategy that helps the ants to identify the shortest distance between the nest (source) and food (destination). These ants walk towards the network by connecting one node after the next node. The transition probability between two hop nodes are expressed as,

$$
t p_{i j}(n)=\left\{\begin{array}{c}
\frac{p h_{i j}^{\gamma}(n) * \mu_{i j}^{\alpha}(n)}{\sum m \epsilon N_{i} p h_{i m}^{\gamma}(n) * \mu_{i m}^{\alpha}(n)}, \text { if } j \in N_{i} \\
0, \text { otherwise }
\end{array}\right.
$$

Where, $t p_{i j}(n)$ represents the transition probability of ant agents walks from one node (i) to another ( $\mathrm{j}$ ) at ' $\mathrm{n}$ ' time interval. $p h_{i, j}$ specifies the pheromone concentration along the path, $\mu_{i, j}$ is the connection status and cost of routing and $N$ intended to be set of organizing the next hop nodes. Finally, $\gamma$ and $\alpha$ indicates pheromone and expected heuristic factor respectively.

At travelling period, the ant marks the path by using pheromone substance. In that way, the other ants travel the same path through the smell. The maximum pheromone holding the path is considered as a shortest path and is chosen as a best path. After then the respective chosen path containing pheromone is updated and is expressed as,

$$
p h_{i j}(n+1)=(1-\varphi) p h_{i j}(n)+\varphi \Delta p h_{i j}(n)
$$


Where, $\Delta p h_{i j}(n)$ indicates the current deposit amount of pheromone in $\mathrm{i}, \mathrm{j}$ nodes on this time, $\varphi$ and $(1-\varphi)$ are the evaporation rate and residual rate of pheromone respectively. And also the range between pheromone value tends to be $[0,1]$.

If there is no movement of ant in the path for a longer time, then the result shows that the path is not in use and is disappear slowly [16]. Figure 1, shows the path finding nature of ant colony optimization technique.
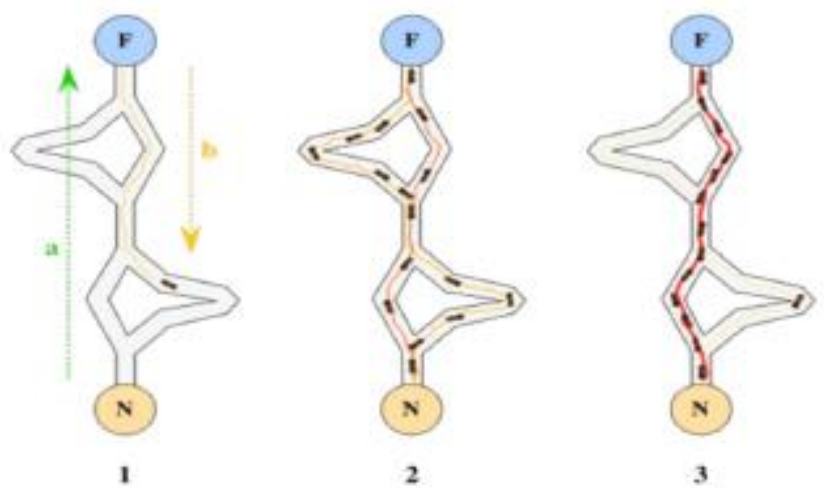

Figure 1.Finding path using ACO technique

\subsection{AntHoc-TBN based routing protocol:}

AntHoc Net is a hybrid architecture inspired by meta-heuristic of ant colony optimization (ACO) algorithm. The routing scheme is constructed for wireless network that exhibit interesting way to route the biological ants by spreading data. In fact, the algorithm such as ABC and AntNet are much desirable for mobile ad-hoc network routing. They are extremely adaptive to route the data via multi-path routing of repeated path. This occurrence can possibly able to cause significant overhead within the network. To address this issue, the MANET used various routing algorithms namely Termite, ARA, AntHoc Net and PERA. An aforesaid routing algorithm examples are comes under Ant Colony routing protocol, especially for route discovery. Among these, AntHoc Net becomes possibly more enthusiastic to minimize overhead because of its routing table. In this research work, the author designed the AntHoc Net with trusted behavioral model and is named as AntHocTBN. The flowchart of this model is depicted in Figure 2.

Generally, the hybrid architecture of AntHoc-TBN model used both the proactive and reactive components. It used two mechanisms of forward ant (FANT) and backward ant (BANT) [17] to discover the route in trusted mode. According to pheromone table, the routing path is set first. It performs some strategy to forward the data packet randomly through various paths. Path selection is the initial process that computes the shortest distance between the nodes. In addition, mobility factor plays a major role in it. Based on the node's movement, the distance computation may vary. Therefore, finding a shortest distance is actually more difficult. So, Dijkstra's algorithm is preferred in this work to calculate the moving node's distance in an effective manner. Let us make a discussion below, 
Dijikstra algorithm, a shortest path analyzer used to find the route of all other nodes from source. It increases its node circulation in extent way to find the shortest path [18]. Distance measure is the main factor that weights the directed graph to find out the shortest path of any neighbour node from the source node. A pseudo code of Dijikstra algorithm is written in stepwise and is shown below,

\section{Pseudo code}

Step 1: Initialize source node's $\left(V_{s}\right)$ distance $\rightarrow 0$ and all other nodes $\rightarrow$ infinity

Step 2: Check the adjacent node for corresponding source node

Step 3: Select the minimum distance node and update the node as visited node

Step 4: If F[ $\left.N_{i}\right]$.Dist $>$ F $\left[\right.$ V].Dist $+S_{v n}$

then $\mathrm{F}\left[N_{i}\right]$.Dist=F[V].Dist $+S_{v n}$ and $\mathrm{F}\left[N_{i}\right]$.path $=V_{s}$

Step 5: Repeat step 3 until the algorithm reaches the shortest path from source to destination

Before initiate the process, the pheromone values become zero. When session starts to communicate, the ant agent performs reactive forward setup of route request to find the next node using Dijikstra algorithm. Based on the distance calculation, the respective source node finds their shortest path and then performs trust mechanism on it. Trustworthiness gives efficient trust value to each neighbor node for secure data communication. Introducing trust based secure model, it continuously monitor the nodes and detect whether there is any black hole attack arise on the node or not. Generally, the behavior of black hole attack is to turn the normal node into malicious node with its hazardous nature. With involving the direct observation, the trust value is compute for each neighbor nodes using Extended Data Routing Information (EDRI) table. This routing table is very helpful for identifying the malicious node and also eliminate it, in the selected path. In previous studies, Data Routing Information (DRI) table is effective to eliminate the malicious node but suffer from process overhead and false positive detection. So the updated version of EDRI is introduced from DRI which is highly efficient to reduce the shortcoming avail in the DRI.

After identified the shortest path, we have to compute the trust for corresponding neighbour node. Trust update is consistently calculated and the updated value is stored in EDRI routing table. So that secure transmission takes place. The condition applied for finding the neighbor node's trust is given by,

$$
\text { Neighbor }{ }_{\text {trust }}=\text { weight } * \text { data forwarded packets }
$$

The weight value is purely decided from user's priority with its empirical way. Meanwhile, the trusted neighbor differentiates the malicious node from normal node based on trust threshold $(\beta)$. If the neighbor trust is less than threshold, then EDRI table set their value as ' 1 '. It means the respective node is concerned with black hole attack so that false behaviour marked on the node to turn it as malicious node. 


$$
E D R I=\left\{\begin{array}{c}
1, \text { if neighbor } \text { trust }<\beta \\
0, \text { otherwise }
\end{array}\right.
$$

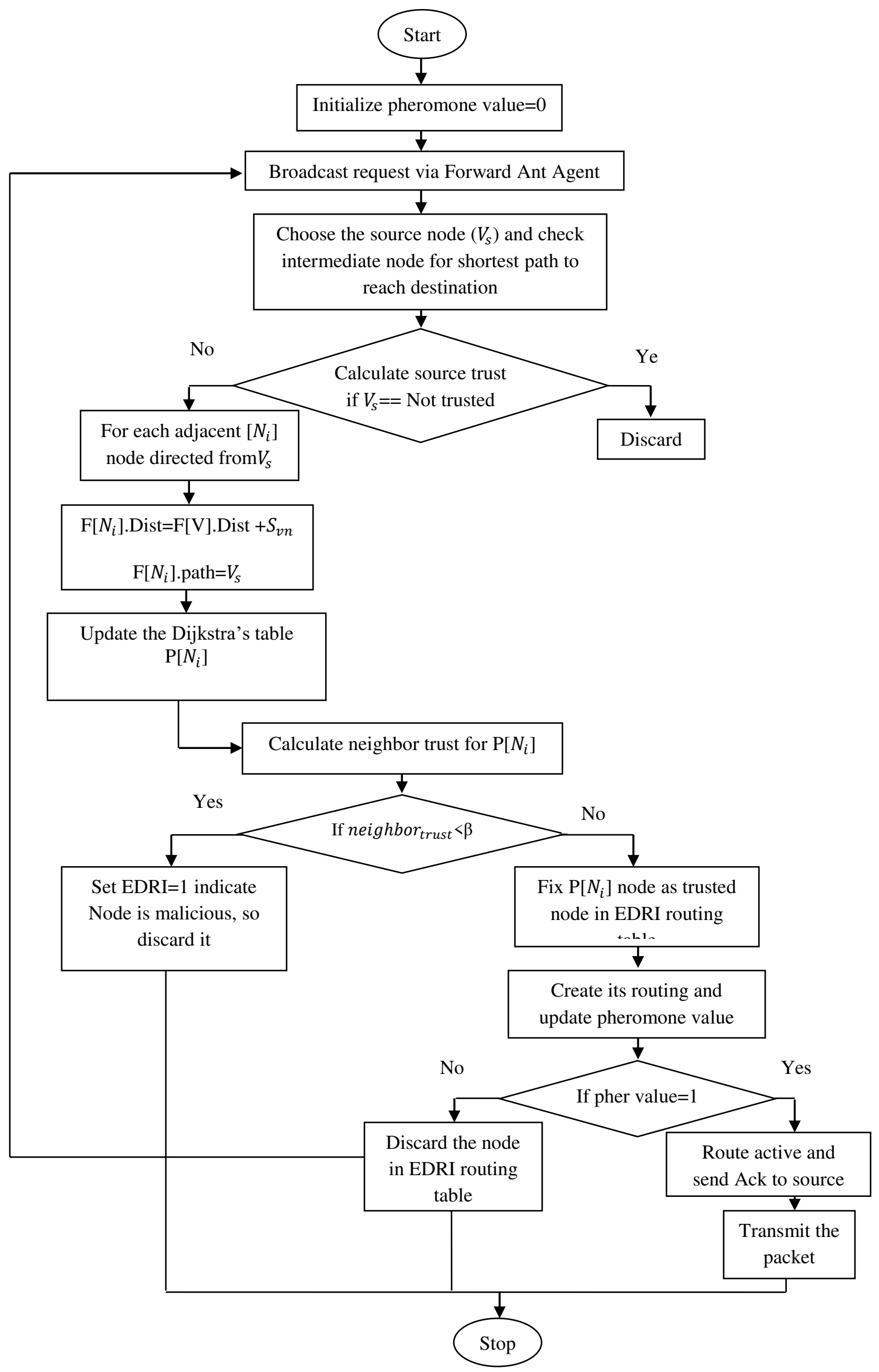




\section{Figure 2. Flowchart of AntHoc based trust behavioural network (AntHoc-TBN) model}

After identified the malicious node, the source node generates sequence number and ID for corresponding malicious node. By receiving this numbers, the EDRI table update the malicious node as black hole node (BHN) and it is mentioned as ' 1 '. Then alert message is broadcast to eliminate the malicious node from the nearby path. In that way, the malicious node gets rid of the routing table.

If the neighbour trust is greater than threshold, then forward ant agent identify the node as trustable node and updated in the EDRI routing table. In this scenario, EDRI table update BHN as ' 0 ' which means there is no black hole attack arises in the corresponding node. The trusted value of each node is updated in EDRI table and its functionality is given by,

$$
1-\text { new }_{\text {trust }}=c\left(1-\text { old }_{\text {trust }}\right)+d\left(1-\text { trust }_{\text {value }(\text { new })}-G\right)
$$

Where, $c$ and $d$ indicates weight of both old and new trust node's value, $G$ is replenishment factor for trust. The variable' $d$ ' is formulated as,

$$
d=c_{1} \times c_{2} \times c_{3}
$$

where,

$$
c_{i}=\frac{\sum_{m a j} w_{i} * \text { trust }_{i}}{w_{m}}
$$

Here, $w_{m}$ depicts the network size, $w_{i}$ represent weight of trusted node and trust $_{i}$ be its trusted value.

Now, the trusted node becomes an active path to update its pheromone value. If value of pheromone becomes ' 1 ', then route become active and send the route response (Ack) to source node. Then the sender proactively transmits the packet to the next node with high trustable one. Else, the same process is continued to compute the shortest distance and trust checking for remaining nodes. The remaining forward ant agent follows the same produce to securely forward the packet until the entire packet reaches the destination.

After reached the destination, the backward ant agents (BANT) receive the acknowledgement message from destination and perform the same procedure to forward that message to source node.

\section{Result and Discussion}

This section evaluates the performance of the proposed mechanism and it is simulated by using NS2 software. This simulator used tool command language (TCL) to implement the network design. The goal of this research work is to eliminate the blackhole attack and make secure data transmission with shortest path distance. To experimentally simulate the proposed work, the technique used 50 nodes to perform operation. In this scenario, the network set the parameters such as channel type of wireless link, the AntHoc Net protocol, simulation area of 
$500 * 500 \mathrm{~m}$, random walk mobility model, 512 bytes of packet size and taken $120 \mathrm{sec}$ of simulation time. A network parameter set up and its corresponding value applied during the simulation as revealed in Table 1.

Table 1. Modelling parameters

\begin{tabular}{|c|c|c|}
\hline S.No & Parameter setup & Values \\
\hline 1. & Simulator & NS2 \\
\hline 2. & Protocol & AntHoc Net \\
\hline 3. & Channel type & 802.11 \\
\hline 4. & Mac layer protocol & $500 * 500 \mathrm{~m}$ \\
\hline 5. & Area of simulation & $120 \mathrm{~s}$ \\
\hline 6. & Mobility model & Random way point \\
\hline 7. & Simulation time & 50 \\
\hline 8. & Packet size & bytes \\
\hline 9. & Number of nodes & \\
\hline
\end{tabular}

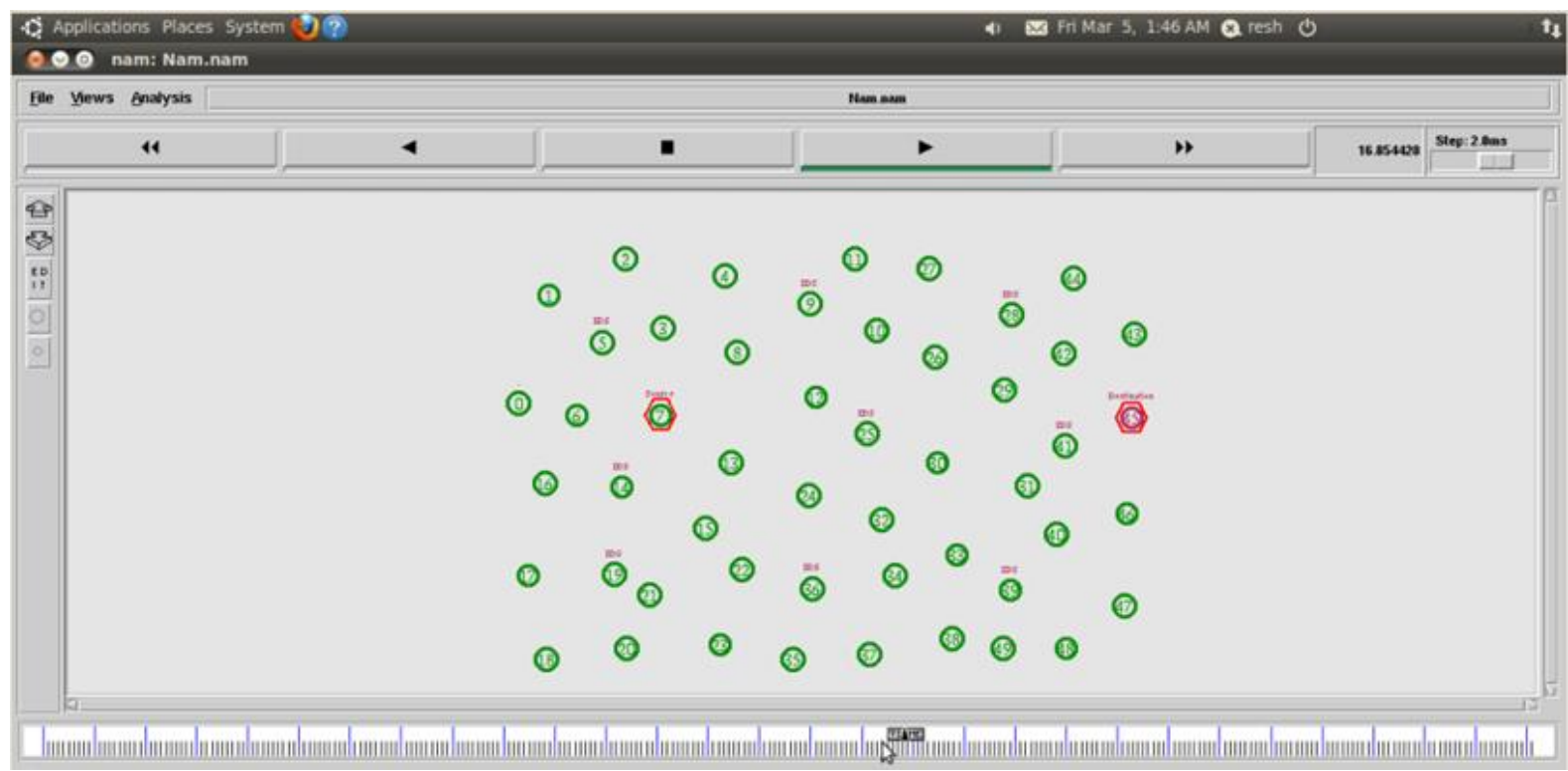

Figure 3. Number of nodes available in simulator area

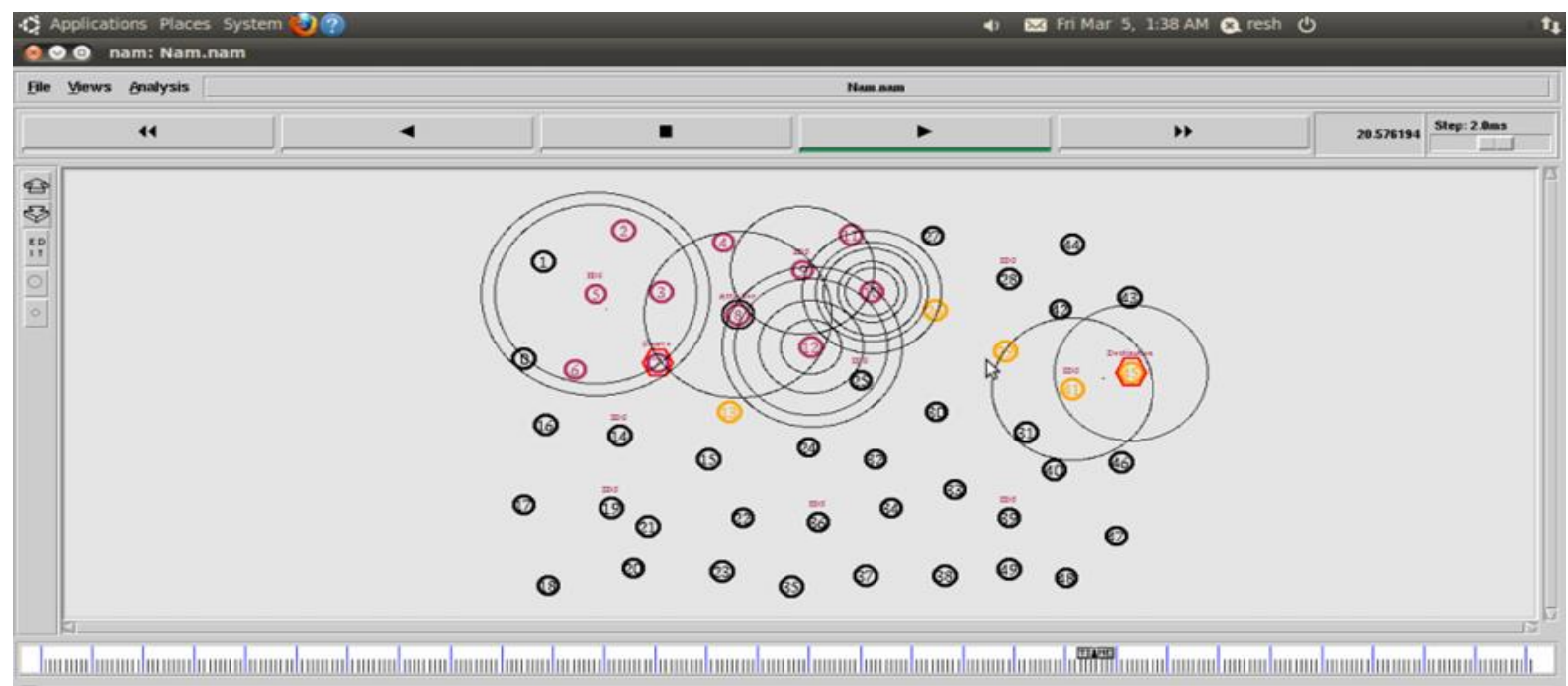




\section{Figure 4. Black hole attack present during routing}

In Figure 3, it shows 50 number of nodes which are randomly distributed in the simulation area. Here, the source and destination are marked by red color and remaining intermediate nodes are kept free. After then, the forward ant agent searches their path using AntHoc Net routing logic. So that, the routing technique imposes dijkstra's algorithm to find shortest path by discover efficient route. By routing the process, the attacker is present within the network and is shown in Figure 4.

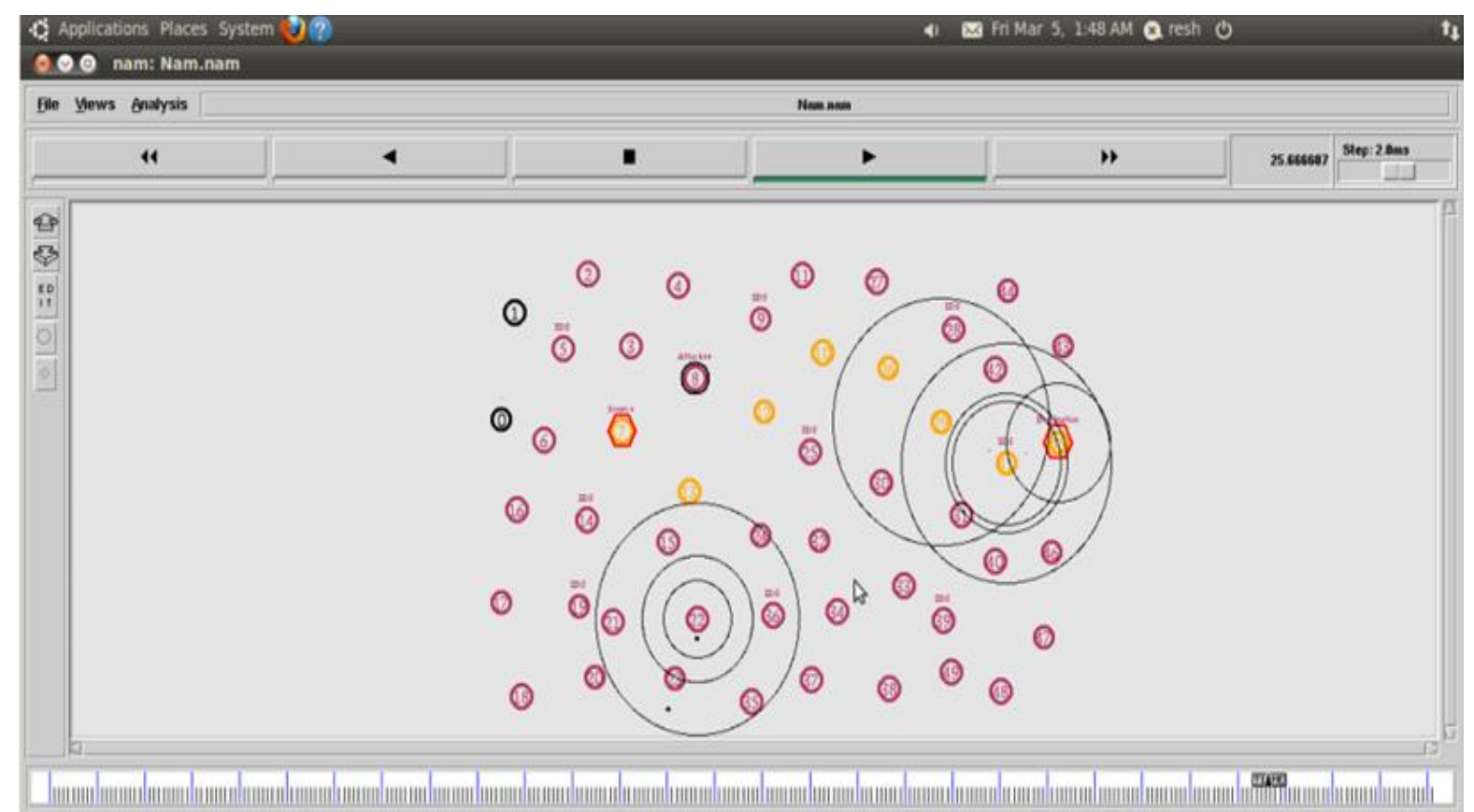

Figure 5. Simulation result of shortest distance along with trusted path using AntHoCTBN model

Trusted node allows efficient and secure data forwarding task with simply reduced the packet loss. It continuously checks the node to identify whether it is trustable node or not. Under simulating the proposed technique, it works very well to effective identify the trusted node in between the corresponding shortest path from source to destination. Simulation result of shortest distance along with trusted path from source to destination as shown in Figure 5.

\subsection{Performance Evaluation}

\section{- Routing Overhead}

Routing overhead is actually meant to add number of routing packets observed from the AntHoc-TBN. The route load in AntHoc-TBN model is comparatively depicts lower result than the existing technique of AODV [21], AntNet [22] and AntHoc Net [23]. In this model, the routing packages arise to compute the routing overhead is route request (RREQ), route response (RREP) and route error (RRER) [19].

$$
\text { Routing Overhead }=R R E P+R R E Q+R R E R
$$


Figure 6, shows the routing overhead (Kbyte) vs node moving speed $(\mathrm{m} / \mathrm{s})$. From $4 \mathrm{~m} / \mathrm{s}$ to $20 \mathrm{~m} / \mathrm{s}$, the routing overhead of AODV, AntNet and AntHoc Net is between 452 to 952, 357 to 850 and 317 to 735 which is somewhat increased. Meanwhile, the AntHoc-TBN improved its routing overhead in between 201 to 637 which is considered to be greater overhead efficiency. High mobility leads to cause high routing overhead because of its route discovery and route failure. Due to routing table behavior, the proposed technique achieves lower routing overhead.

Figure 7, shows the routing overhead versus no. of malicious nodes. Out of 50 nodes, five nodes are a malicious node which is greatly affected by blackhole attack. From the network, the routing overhead of AODV, AntNet, AntHoc Net and proposed model are insists with five malicious nodes. The result shows that the proposed technique achieves good routing overhead as compared to remaining models.

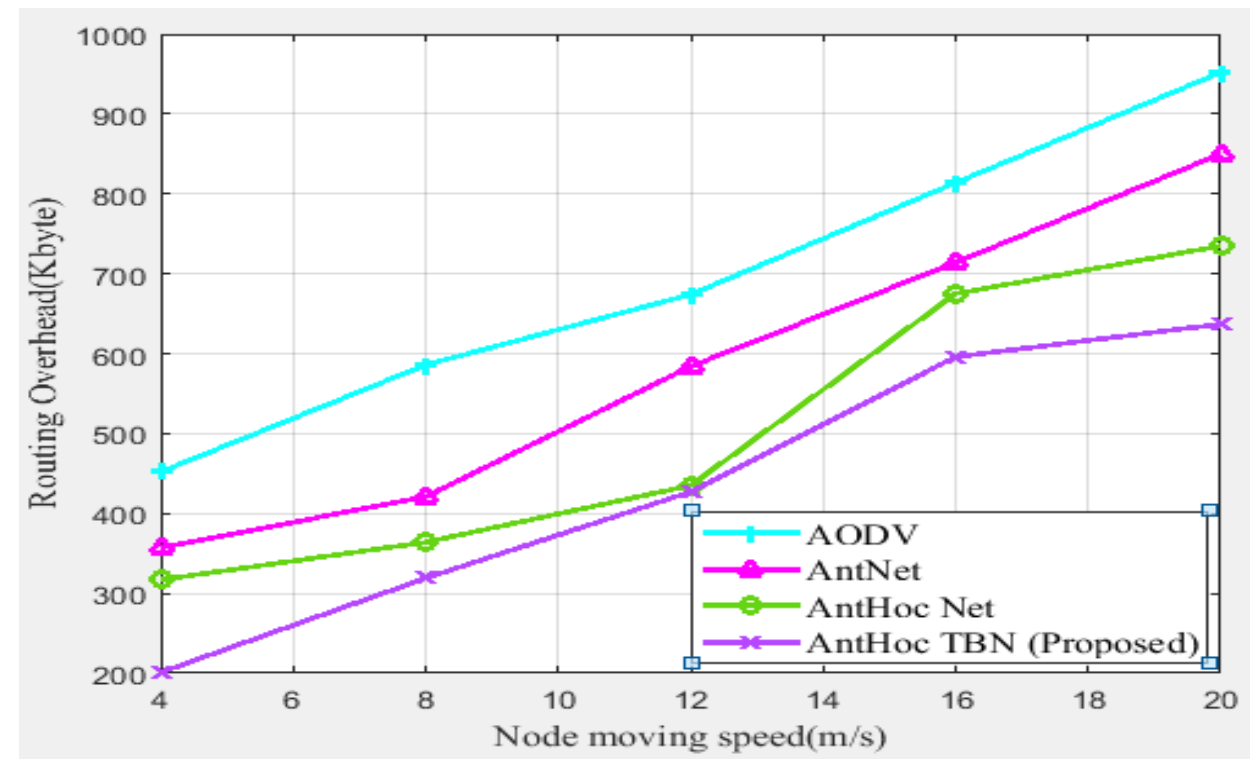

Figure 6. Routing overhead versus node moving speed in $\mathrm{m} / \mathrm{s}$

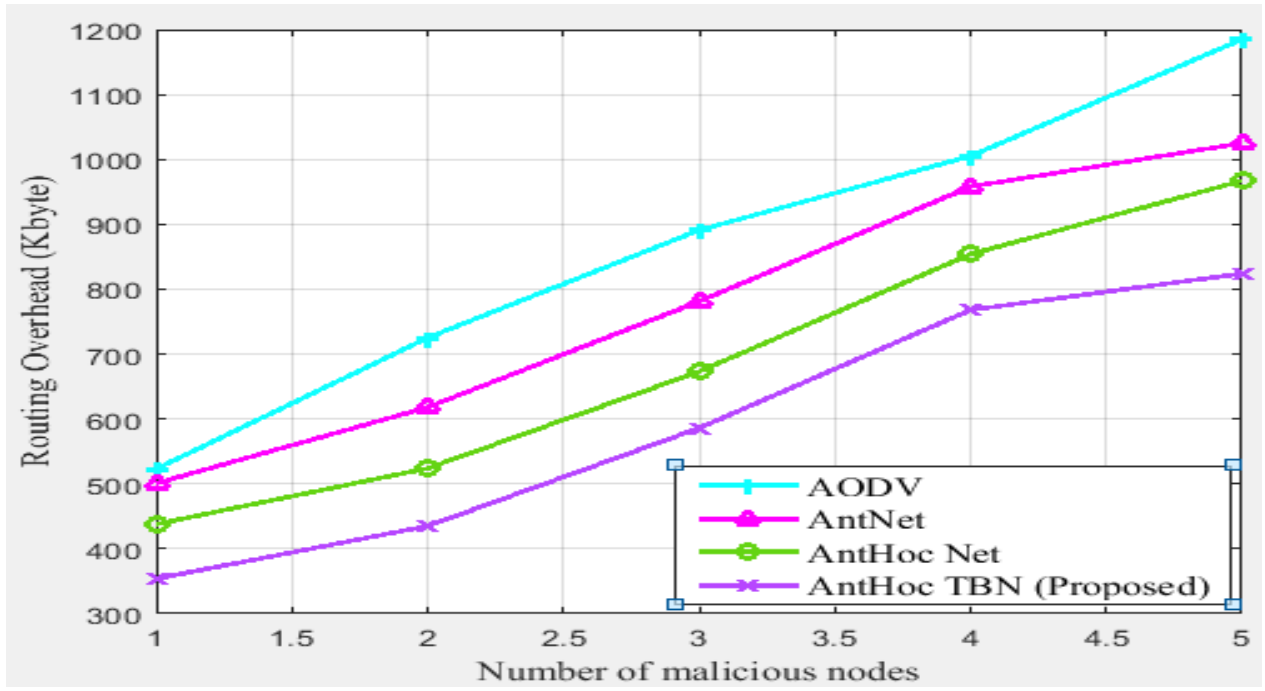

Figure 7. Routing overhead versus no. of affected node 


\section{- Delay}

Delay is the time difference observed from source to destination during packet transfer [19]. The delay is much familiar to connect several factors such as extreme load and connection point's distance. If the network has larger excessive load and longer connection point's distance, then automatically delay attains a maximum value. The maximum delay value may not provide better quality for the network. So the delay needs to be reduced in lower value.

$$
\text { Delay }=\frac{\text { Received packet duration-Sent packet duration }}{\text { Total of received package }}
$$

Figure 8 , shows the delay effectiveness versus node moving speed $(\mathrm{m} / \mathrm{s})$. For $4 \mathrm{~m} / \mathrm{s}$ of node mobility, the AntHoc-TBN model achieves 0.058 which is considered to be a least delay value among the remaining models. Therefore, the experiment concludes that least delay value can appropriately improve the system with greater effectiveness.

It shows the delay effectiveness of different routing techniques such as AODV, AntNet, AntHoc Net and AntHoc-TBN as shown in Figure 9. Under circumstance, the routing protocols rediscover the path from malicious node which leads to cause latency in the network. Possibly comparing the routes from different strategies of protocols, they may rediscover the route with varying time delay. When comparing the delay efficiency against five malicious nodes, the proposed scheme achieves a delay of $0.153 \mathrm{~s}, 0.166 \mathrm{~s}, 0.179 \mathrm{~s}, 0.19 \mathrm{~s}$ and 0.185 s respectively. Comparably, the obtained value shows much lower result than the other techniques.

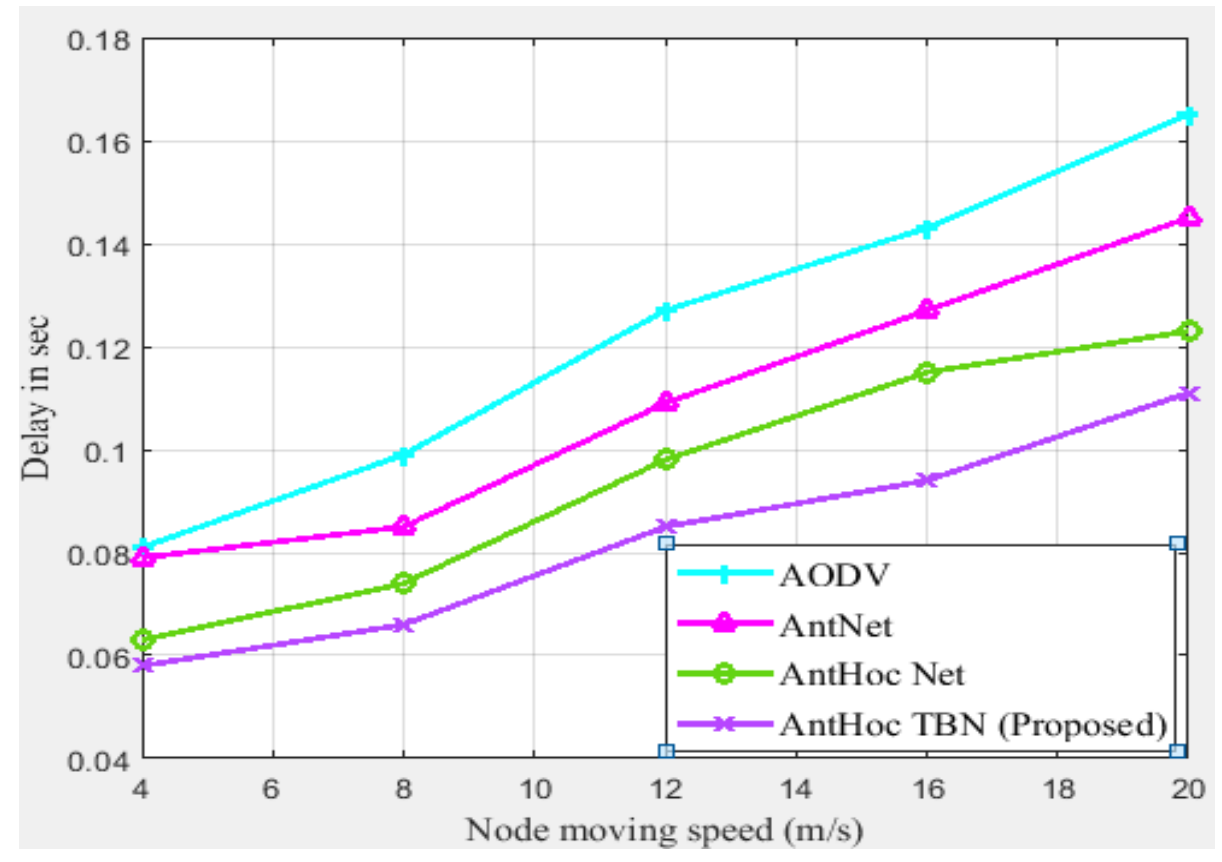

Figure 8. Delay versus node moving speed in $\mathrm{m} / \mathrm{s}$ 


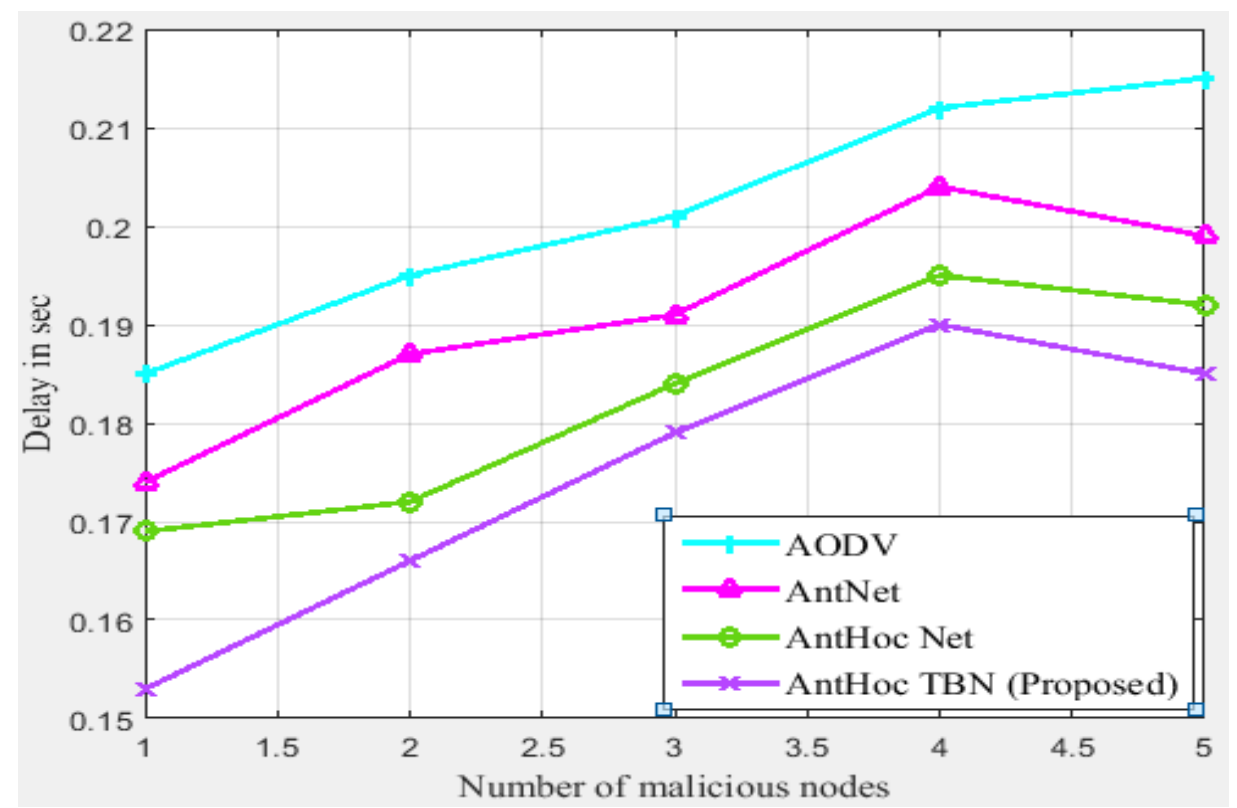

Figure 9. Delay versus number of malicious node

\section{- Packet Loss}

It is defined as the ratio of subtracting the total number of sent and receive packet over total packet sent [19]. Some factors such as packet corruption, buffer overhead, network overhead, network error rate etc., are greatly affect the network to cause packet loss problem. To compute packet loss, the expression is given by,

$$
\text { Packet Loss }=\frac{\text { total no.of sent packet-total no.of received packet }}{\text { total no.of sent packet }}
$$

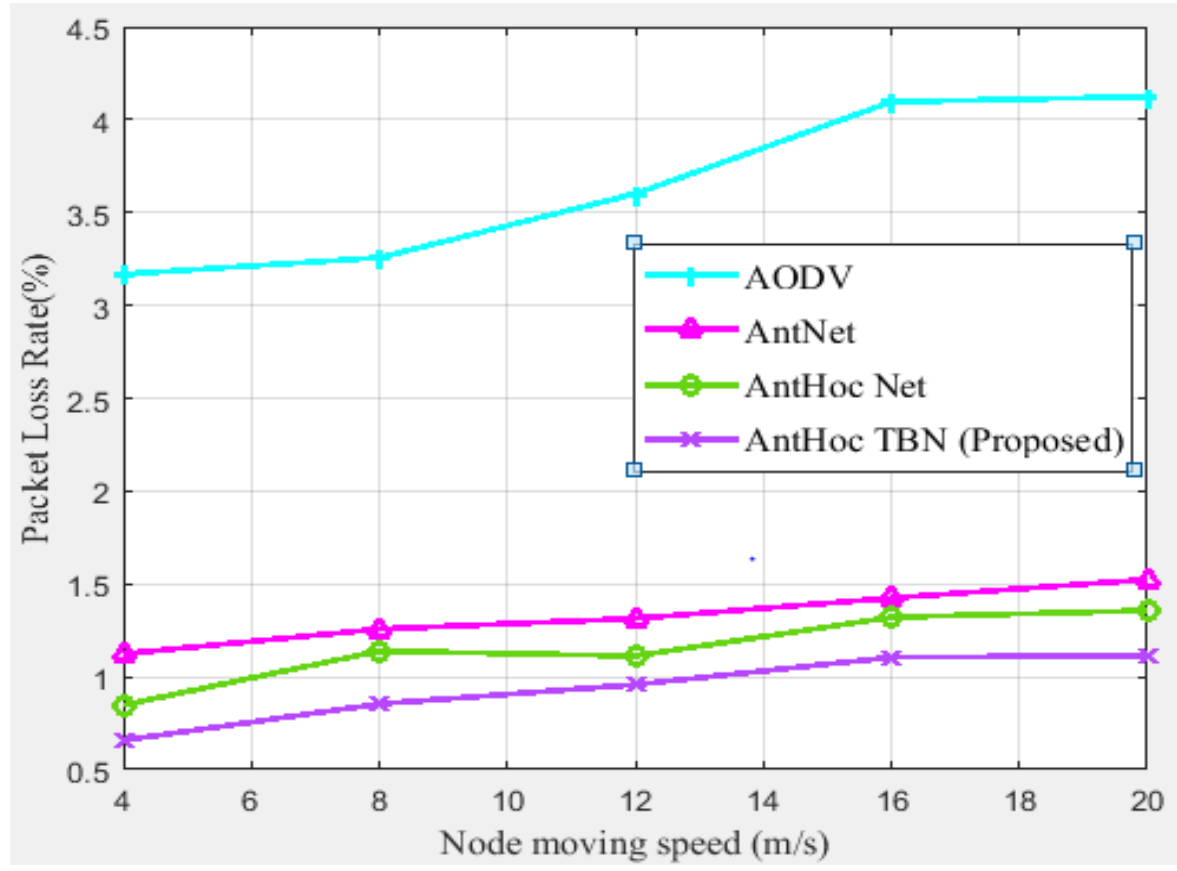

Figure 10. Packet loss versus node moving speed 
Figure 10, shows packet loss values versus node moving speed. When node varies, it periodically drops its packet ratio at certain time interval. AODV protocol occupies highest amount of packet loss which is exactly 3.649 of mean value. The proposed approach attains improved packet loss ratio while deriving the mobile ad hoc network. The mean value of AODV, AntHoc, AntHoc Net and AntHoc-TBN (Proposed) shows 3.649, 1.328, 1.155 and 0.937 respectively. Comparatively analysed the result of packet loss ratio, the proposed shows much reduced loss than the existing techniques of AODV, AntNet and AntHoc Net.

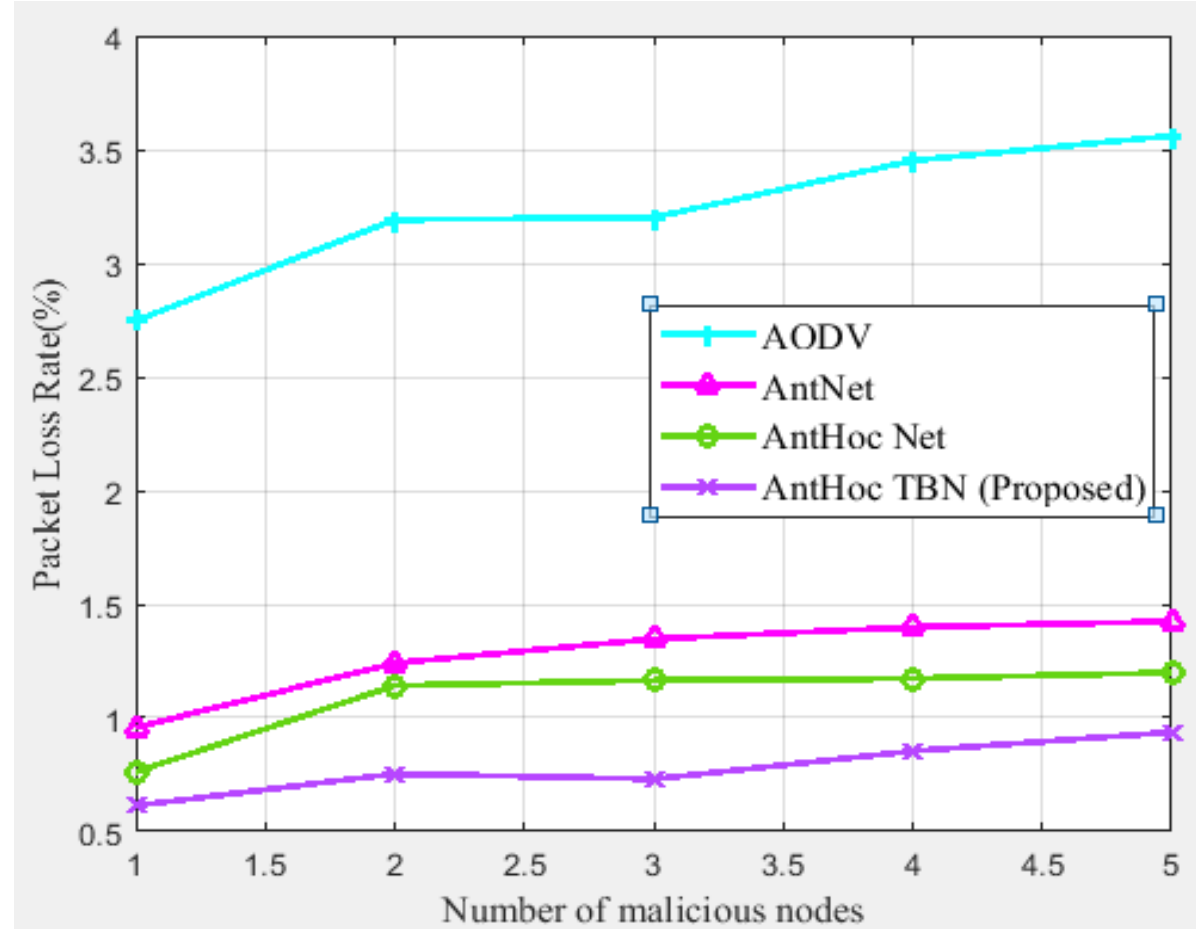

Figure 11. Packet loss versus number of malicious node

Figure 11, shows packet loss versus number of malicious nodes. Due to increasing the malicious node, the packet loss also increased further. The analysis process is carried at $20 \mathrm{~m} / \mathrm{s}$ of node's mobility. For single malicious node, AODV routing protocol has 2.74 value of packet loss and it is increases sharply to 3.56 when remaining malicious nodes are carried within the network. As compared the packet loss mean value efficiency against no. of malicious node, the proposed, AntHoc Net, AntNet and AODV routing protocol achieves $0.778,1.088,1.273$ and 3.23 respectively. To experimentally proves that the proposed attains greater packet loss efficiency.

\section{- Packet delivery ratio}

It is defined as a ratio of calculating total number of collected packet by ending point to the total transmitted packet from starting point [20] and is expressed by,

$$
P D R=\frac{\text { total no.of received packet }}{\text { total no.of sent packet }}
$$


The following figure 12, shows different node movement analysis for various routing protocols which are experimentally performed to establish packet delivery result. The proposed scheme produce much better PDR values of 96.1\%,96\%, 95.9\%, 94.7\% and $93.8 \%$ for different node mobility of $4 \mathrm{~m} / \mathrm{s}, 8 \mathrm{~m} / \mathrm{s}, 12 \mathrm{~m} / \mathrm{s}, 16 \mathrm{~m} / \mathrm{s}$ and $20 \mathrm{~m} / \mathrm{s}$ respectively. This obtained value is considered as far improved result over the remaining schemes. Considered $4 \mathrm{~m} / \mathrm{s}$ of node mobility and is evidentially proved that the proposed technique increases its PDR value by $3.12 \%, 8.94 \%$ and $21.1 \%$ of AntHoc Net, AntNet and AODV routing techniques respectively.

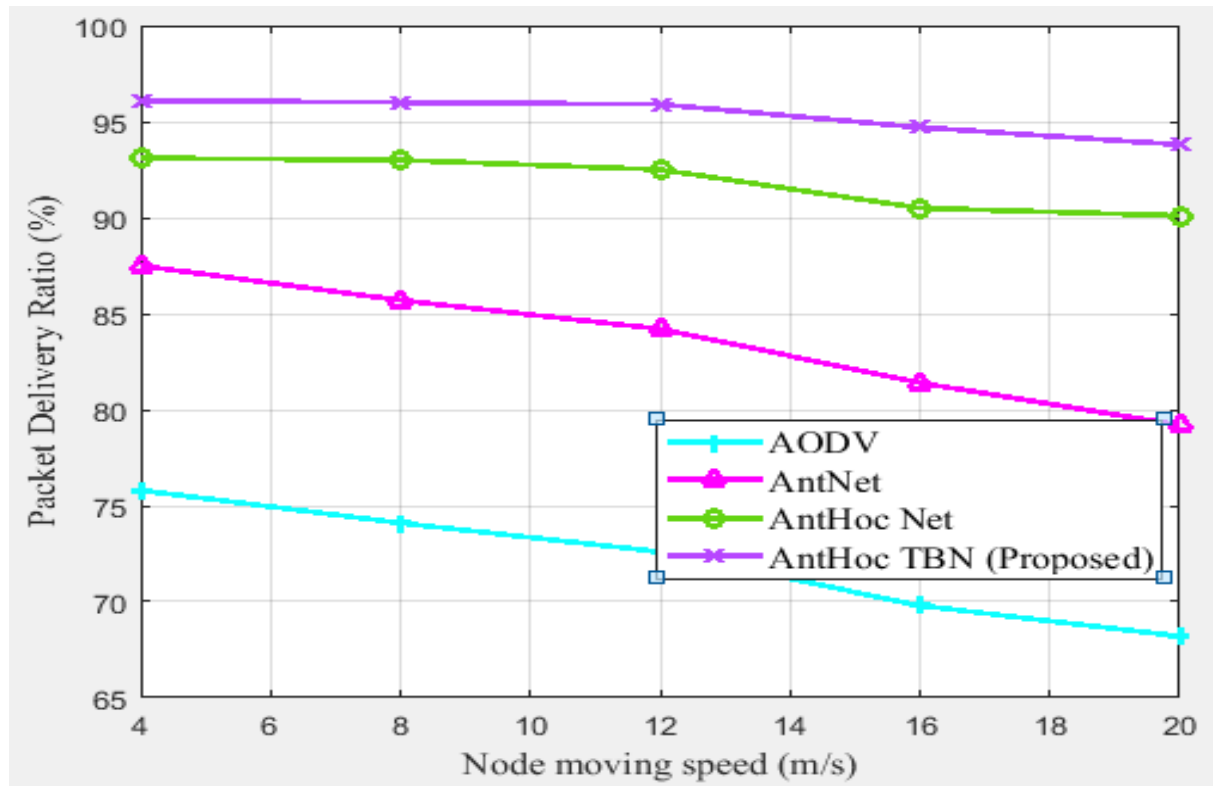

Figure 12. Packet delivery ratio versus node moving speed

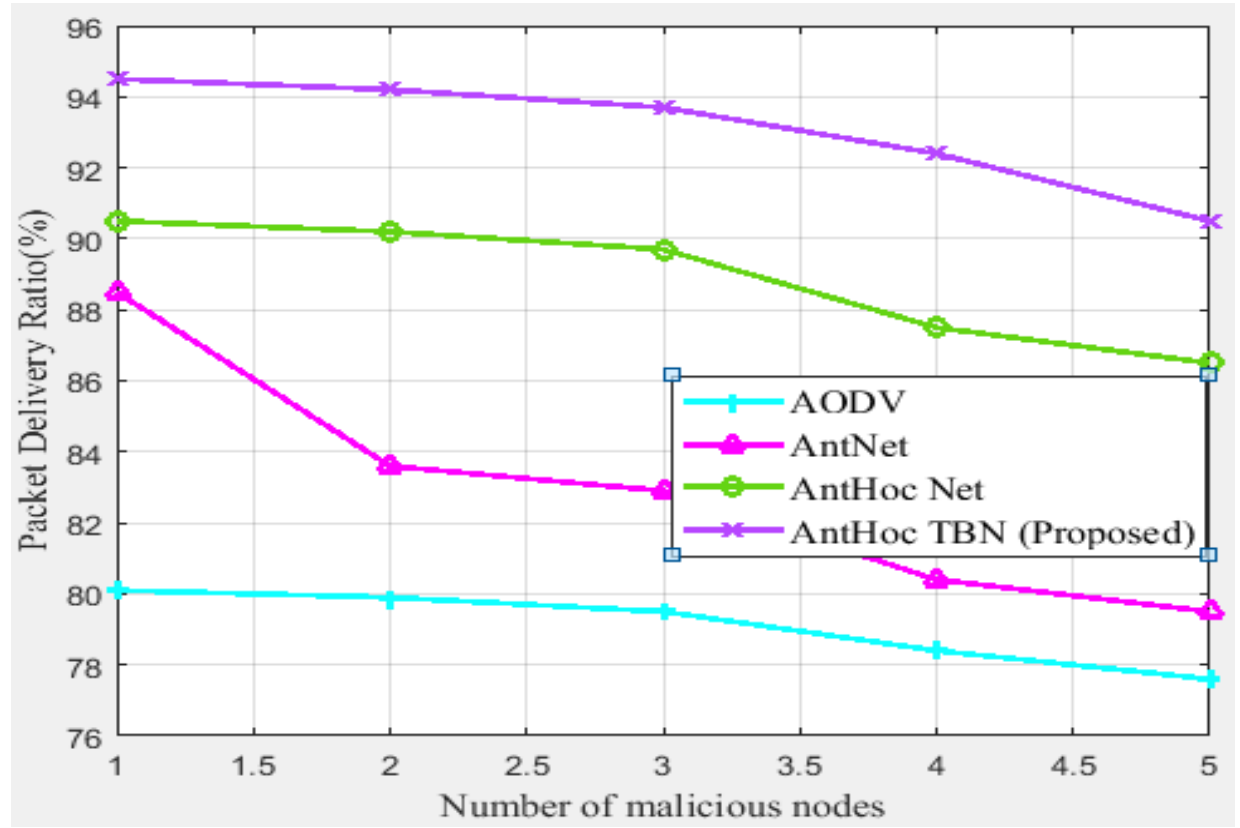

Figure 13. Packet delivery ratio versus malicious node 
Another comparison chart explains the PDR percentage with respect to no. of malicious node and is shown in Figure 13. Among different routing protocols, the AntHocTBN model leads a greater efficiency because the technique is much familiar to choose another path immediately whether there is any misbehaving node occurs. For single malicious node, the proposed work improves its PDR value by $4.23 \%, 6.34 \%$ and $15.23 \%$ of AntHoc Net, AntNet and AODV routing techniques respectively.

\section{Conclusion}

Mobile ad hoc network is an open network that can easily affected by various attacks. Possibly affected attack in MANET is black hole attack which cause severe degradation in network performance. To maintain the network to be effective, this paper proposes an AntHoc based trust behavioural network (AntHoc-TBN) model which is much satisfied to discover the route with trusted basis. The proposed technique analyzes the performance and shows their effectiveness. To conclude the work, we have to observe the packet delivery ratio in terms of node mobility and no. of malicious attack. It shows that proposed scheme produce much better PDR values of $96.1 \%, 96 \%, 95.9 \%, 94.7 \%$ and $93.8 \%$ for different node mobility of $4 \mathrm{~m} / \mathrm{s}, 8 \mathrm{~m} / \mathrm{s}, 12 \mathrm{~m} / \mathrm{s}, 16 \mathrm{~m} / \mathrm{s}$ and $20 \mathrm{~m} / \mathrm{s}$ respectively. For $4 \mathrm{~m} / \mathrm{s}$ of node mobility and single malicious node, it is evidentially proved that the proposed technique increases its PDR value by $3.12 \%, 8.94 \%$ and $21.1 \%$ and $4.23 \%, 6.34 \%$ and $15.23 \%$ of AntHoc Net, AntNet and AODV routing techniques respectively.

\section{Declarations}

Ethical Approval : All the authors mentioned in the manuscript have agreed for authorship, read and approved the manuscript, and given consent for submission and subsequent publication of the manuscript.

Consent to Participate : Not applicable for this section

Consent to Publish : Not applicable for this section

Authors Contributions: M Karthika analyzed and interpreted the data regarding the secure route finding. M Rajkumar was a major contributor in writing the manuscript. All authors read and approved the final manuscript.

Funding: There is no funding source.

Conflict of Interest: The authors declare that they have no conflict of interest.

Availability of data and materials : We recognise it is not always possible to share research data publicly, for instance when individual privacy could be compromised, and in such instances data availability should still be available in the manuscript. 


\section{REFERENCES}

[1] RadhikaRanjan Roy, "Handbook of Mobile Ad Hoc Networks for Mobility Models",Springer: New York, NY, USA, ISBN 978-1-4419-6048-1, 2011.

[2] Radha Raman Chandan, and Pramod Kumar Mishra, "A Review of Security Challenges in Ad-Hoc Network", International Journal of Applied Engineering Research 13, no. 22, pp. 16117-16126, 2018.

[3]Vishnu Balan E, Priyan MK, Gokulnath C, Usha Devi G, "Fuzzy based intrusion detection systems in MANET", Procedia Comp Sci, Elsevier, vol. 50, pp. 109 - 114, 2015

[4] HariharanRajadurai, and Usha Devi Gandhi, "Fuzzy based collaborative verification system for Sybil attack detection in MANET", Wireless Personal Communications, vol. 110, no. 4, pp. 2179-2193, 2020.

[5] Anil Kumar, R. S. Shukla, and R. K. Shukla. "Simulation Based Comparative Study of Routing Protocols in MANET", Invertis Journal of Renewable Energy, vol.10, no. 1, pp. 4248, 2020.

[6] Deepika Kukreja, S. K. Dhurandher, B. V. R. Reddy, "Power aware malicious nodes detection for securing MANETs against packet forwarding misbehavior attack", J Ambient Intell Human Comput, 2017.

[7] K. Prabha, "Performance assessment and comparison of efficient ad hoc reactive and proactive network routing protocols", SN Computer Science, vol.1, no. 1, pp. 1-7, 2020.

[8]FaïzaTabbana,"Performance Comparison and Analysis of Proactive, Reactive and Hybrid Routing Protocols for Wireless Sensor Networks", International Journal of Wireless \& Mobile Networks (IJWMN), vol.12, 2020.

[9] Salem A.Almazok, and Bülent Bilgehan, "A novel dynamic source routing (DSR) protocol based on minimum execution time scheduling and moth flame optimization (METMFO)", EURASIP Journal on Wireless Communications and Networking, no. 1,pp. 1-26, 2020.

[10] Ghassan A. Qasmarrogy, and Yazen S. Almashhadani, "Ad Hoc On-demand Distance Vector Inherent Techniques Comparison for Detecting and Eliminating the Black Hole Attack Nodes in Mobile Ad Hoc Network", Cihan University-Erbil Scientific Journal, vol. 4, no. 1, pp. 77-81, 2020.

[11] Sina Shahabi, Ghazvini, M., \&Bakhtiarian, M, (2016), "A modified algorithm to improve security and performance of AODV protocol against black hole attack", Wireless Networks, vol. 22, no. 5, pp. 1505-1511, 2016.

[12] Dost Muhammad Khan, TalalAslam, Nadeem Akhtar, Salman Qadri, Imran Mujaddid Rabbani, and Muhammad Aslam, "Black Hole Attack Prevention in Mobile Ad-hoc Network 
(MANET) Using Ant Colony Optimization Technique", Information Technology and Control, vol.49, no. 3, pp. 308-319, 2020.

[13] Ephantus Gichuki Mwangi, Geoffrey M. Muketha, and Gabriel N. Kamau, "Optimized Trust-Based DSR Protocol to Curb Cooperative Blackhole Attacks in MANETs Using NS3", International Journal of Networks and Communications 2020, vol.10, no.1, pp. 10-19, 2020 .

[14] D. Mishra, Arukonda. S, "Black Hole Attack Prevention Techniques in MANET: A Review", International Journal of Engineering and Computer Science, vol. 3, no. 6, pp. 6735-6738. ISSN: 2319-7242, 2014.

[15] I.S. Imad, Majdi, Z. R, "Various Solutions of Black Hole Attack in mobile Ad Hoc Network (MANET)" International Journal of Computer Science and Information Security, vol.12, no.8, 2018.

[16] Manjula Poojary, and B. Renuka, "Ant colony optimization routing to mobile ad hoc networks in urban environments", International Journal of Computer Science and Information Technologies, vol. 2, no. 6, pp. 2776-2779, 2011.

[17] Ajeng Mayang, and Savitri Galih, "Comparative Performance AODV-ANT and AOMDV-ANT in BlackHole, "International Journal of Engineering \& Technology 7, no. 4.34, pp. 358-363, 2018.

[18] Dhanalakshmi Natarajan, and Alli P. Rajendran, "AOLSR: hybrid ad hoc routing protocol based on a modified Dijkstra's algorithm", EURASIP Journal on Wireless Communications and Networking 2014, no. 1, pp. 1-10, 2014.

[19] Tri Kuntoro Priyambodo, Danur Wijayanto, and Made Santo Gitakarma, "Performance Optimization of MANET Networks through Routing Protocol Analysis", Computers, vol.10, no.1, 2021.

[20] Madhvi Patel, and Dileep Sharma, "Performance evaluation of artificial ant colony using zone based routing approach in MANET", 2017.

[21] S. Sankara Narayanan, and G. Murugaboopathi, "Modified secure AODV protocol to prevent wormhole attack in MANET", Concurrency and Computation: Practice and Experience, vol. 32, no. 4, e5017, 2020.

[22] Giamni Di Caro and Marco Dorigo, "AntNet: a mobile agents approach to adaptive routing”, Technical Report IRIDIA, pp. 1-27, 1997.

[23] B. Kalaavathi, K. Duraiswamy, "Ant Colony Based Node Disjoint Hybrid Multi-path Routing for Mobile Ad Hoc Network", Journal of Computer Science, vol. 4, no. 2, pp. 80-86, 2008. 
Figures

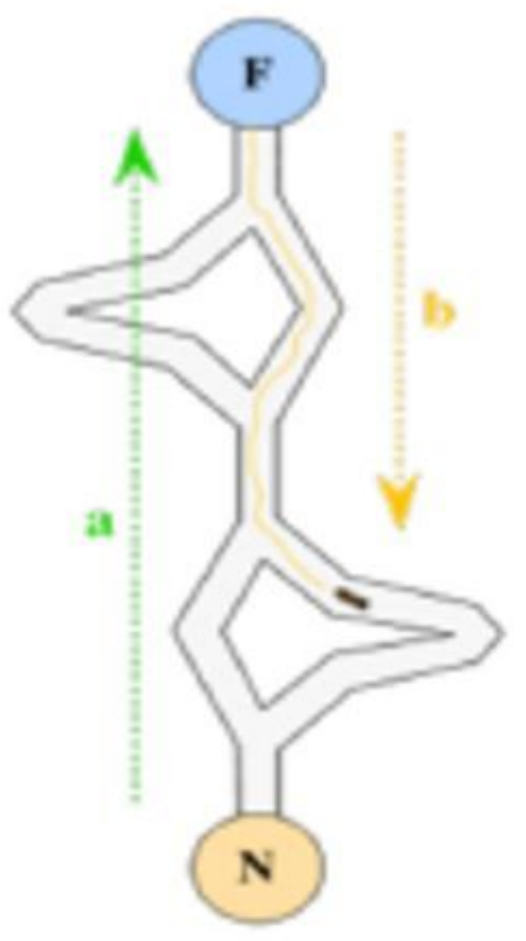

1

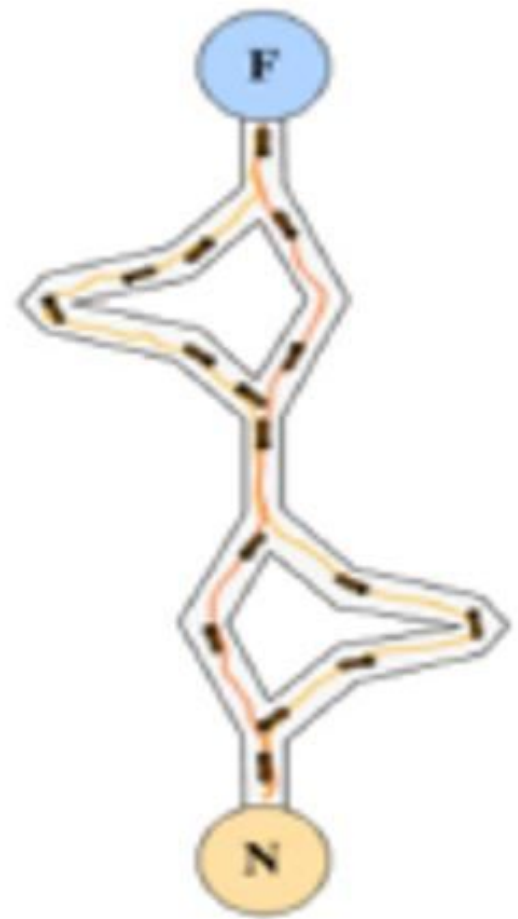

2

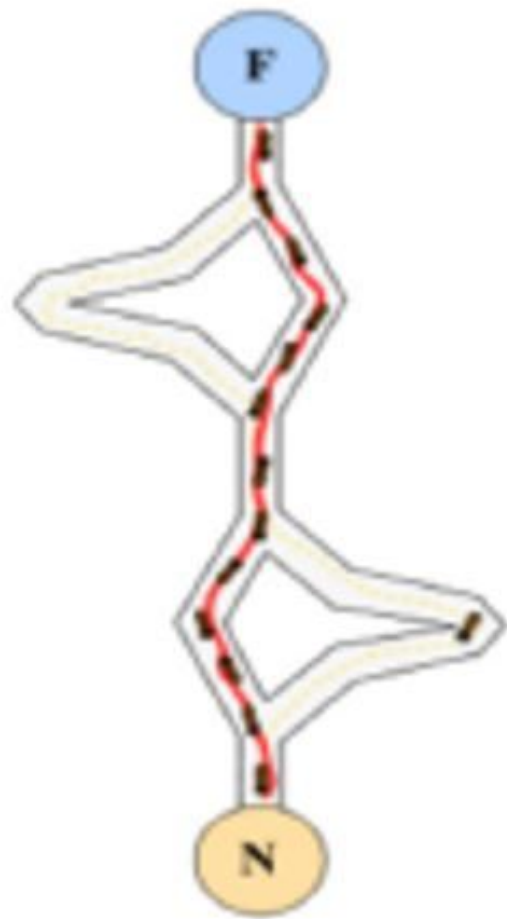

3

Figure 1

Finding path using $\mathrm{ACO}$ technique 


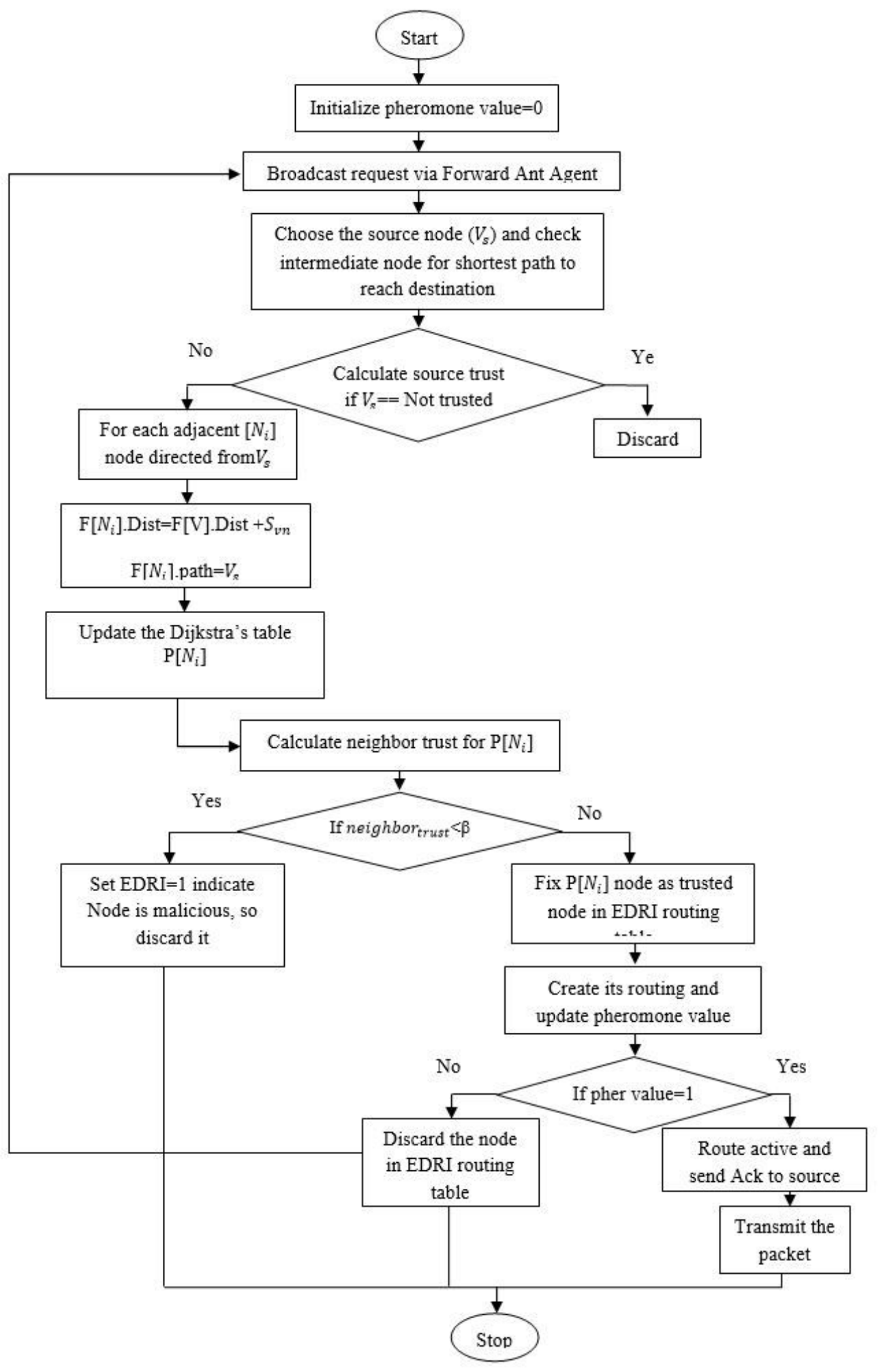

Figure 2

Flowchart of AntHoc based trust behavioural network (AntHoc-TBN) model 
QO nam: Nam.nam

Ele Vews Gnalysis

tham ane

\begin{tabular}{|c|c|c|c|c|c|c|}
\hline 14 & 4 & a & 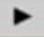 & 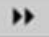 & 16 astea & Stev: 2 Ams \\
\hline
\end{tabular}

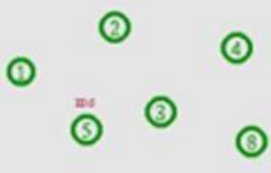
$\odot_{0}^{\circ} 0$
(1)
$\odot \odot \div$
○
๑ี

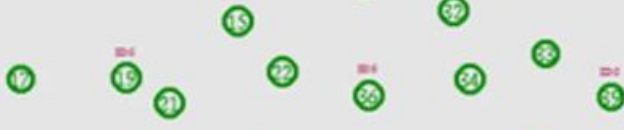
(1)
๑)

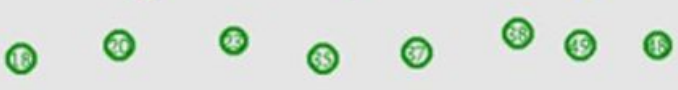

|

Figure 3

Number of nodes available in simulator area

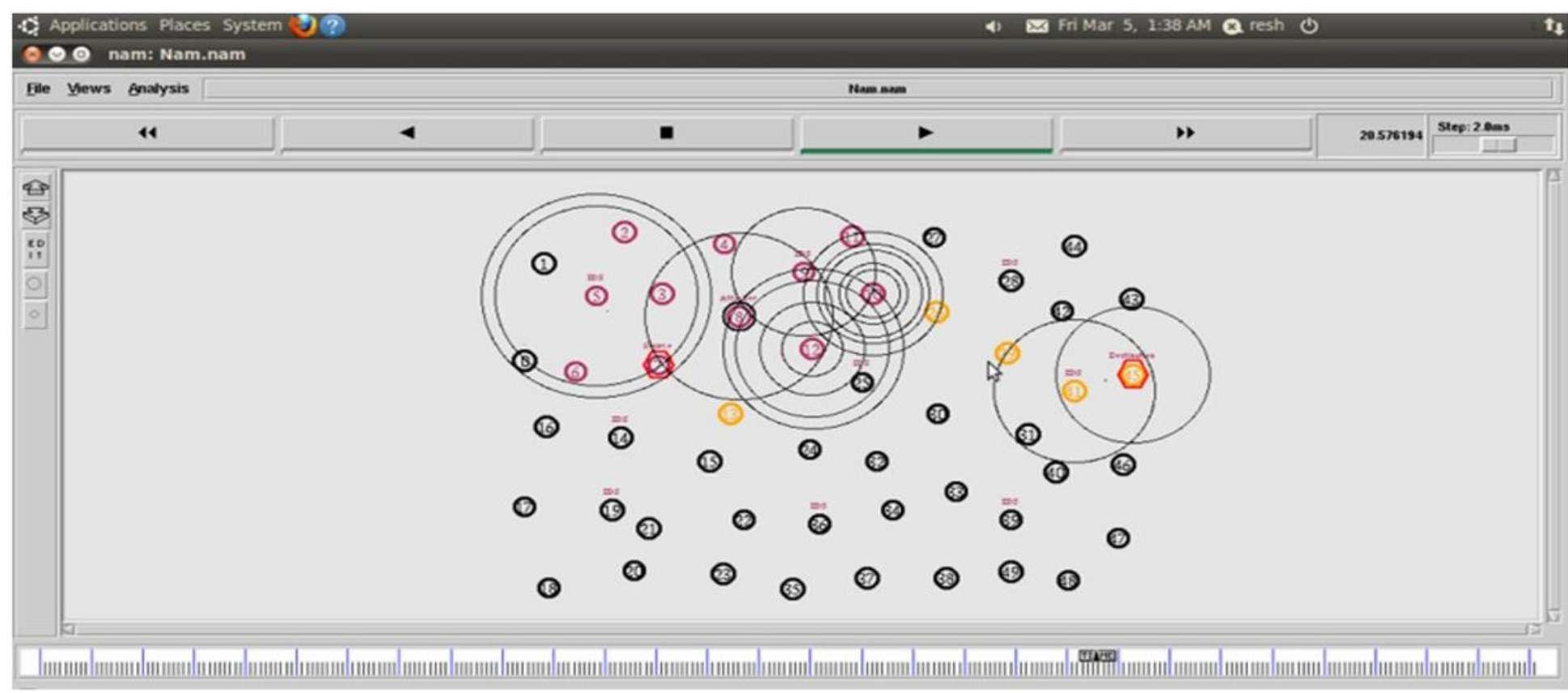

Figure 4

Black hole attack present during routing 


\section{QQO nam: Nam.nam}

Flle Mews Bnalysis

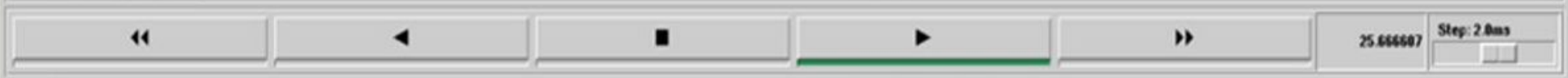

\begin{tabular}{|l}
\hline 8 \\
8 \\
\hline 10 \\
\hline 10 \\
\hline 0
\end{tabular}

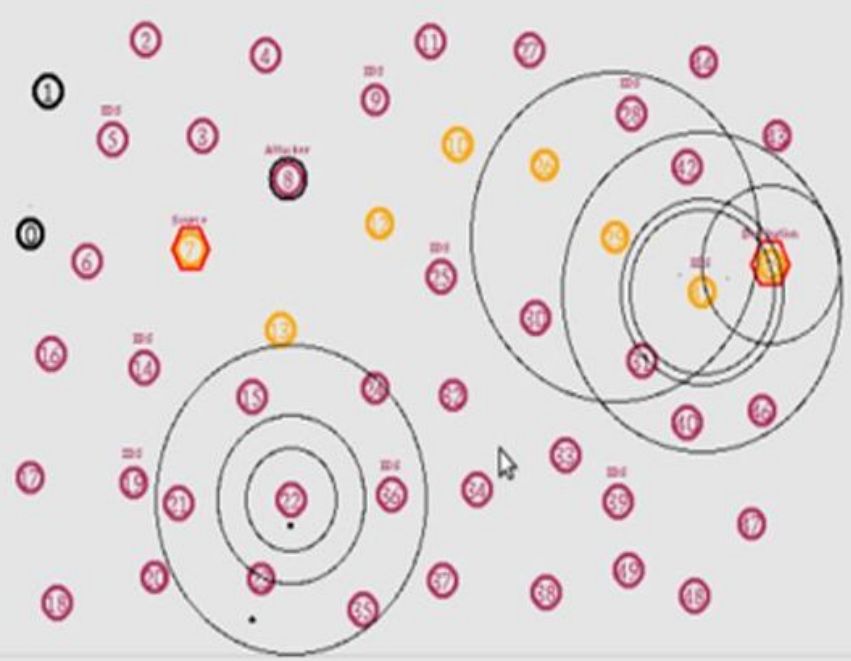

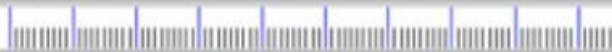

Figure 5

Simulation result of shortest distance along with trusted path using AntHoC-TBN model

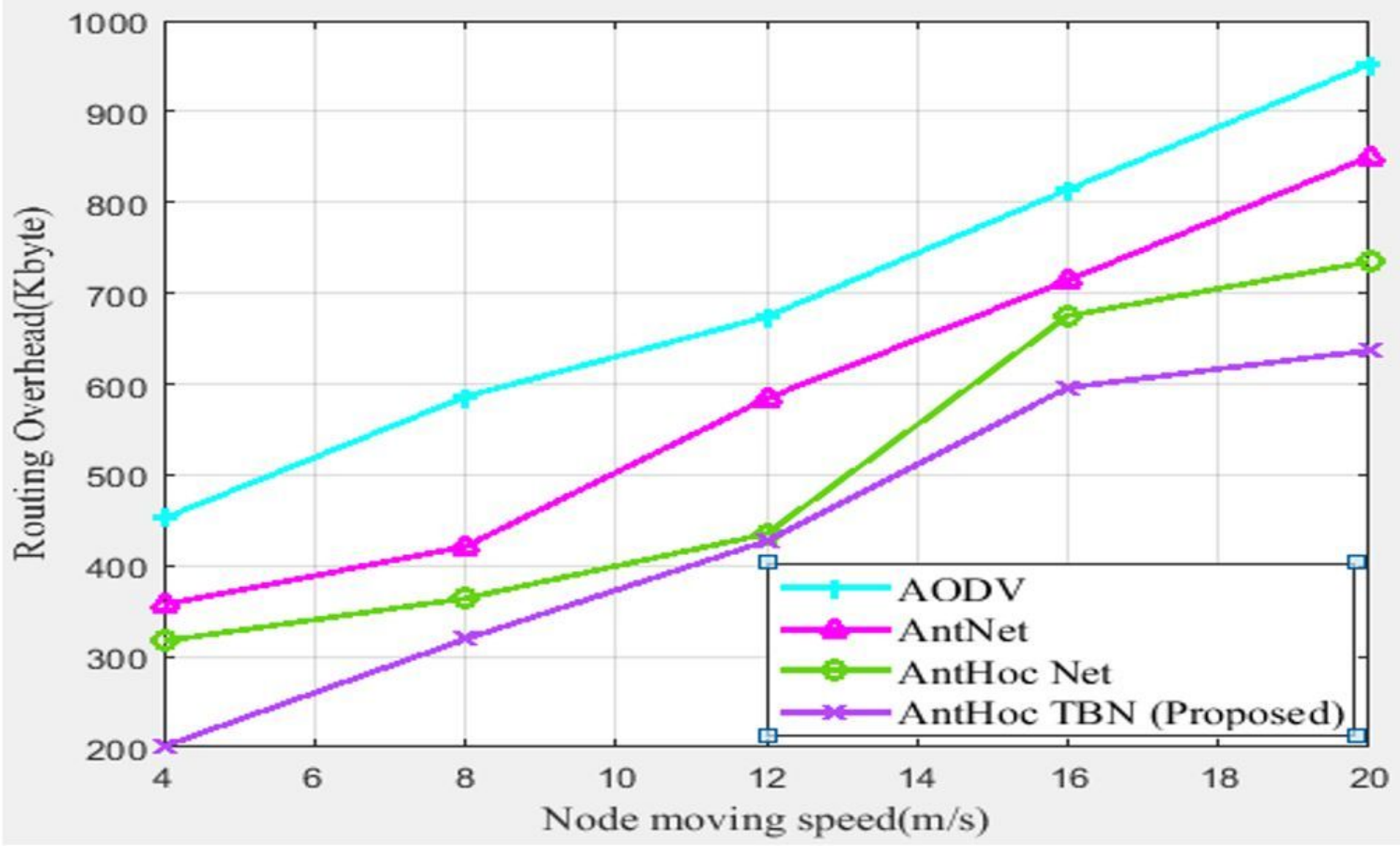


Figure 6

Routing overhead versus node moving speed in $\mathrm{m} / \mathrm{s}$

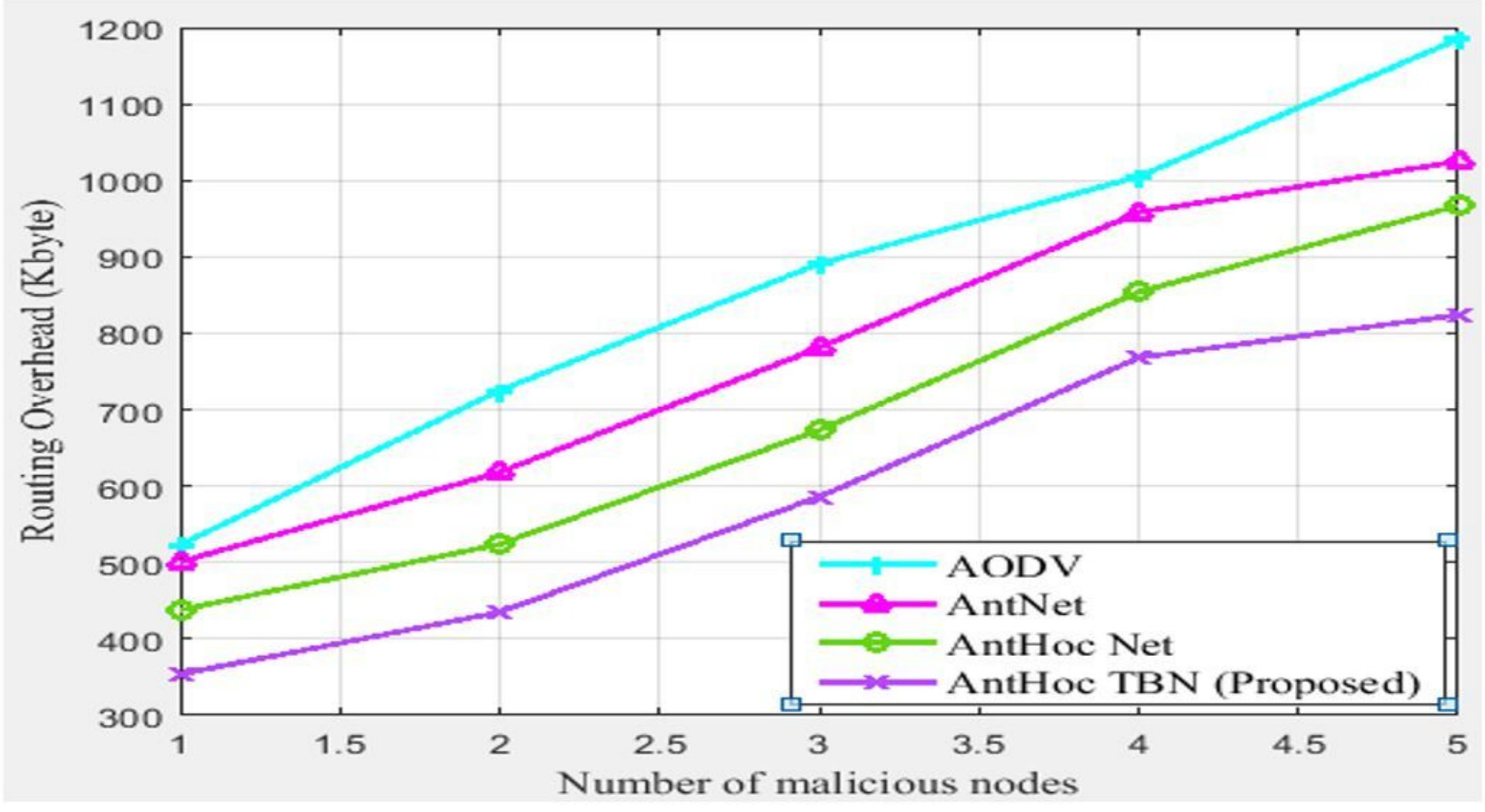

Figure 7

Routing overhead versus no. of affected node 


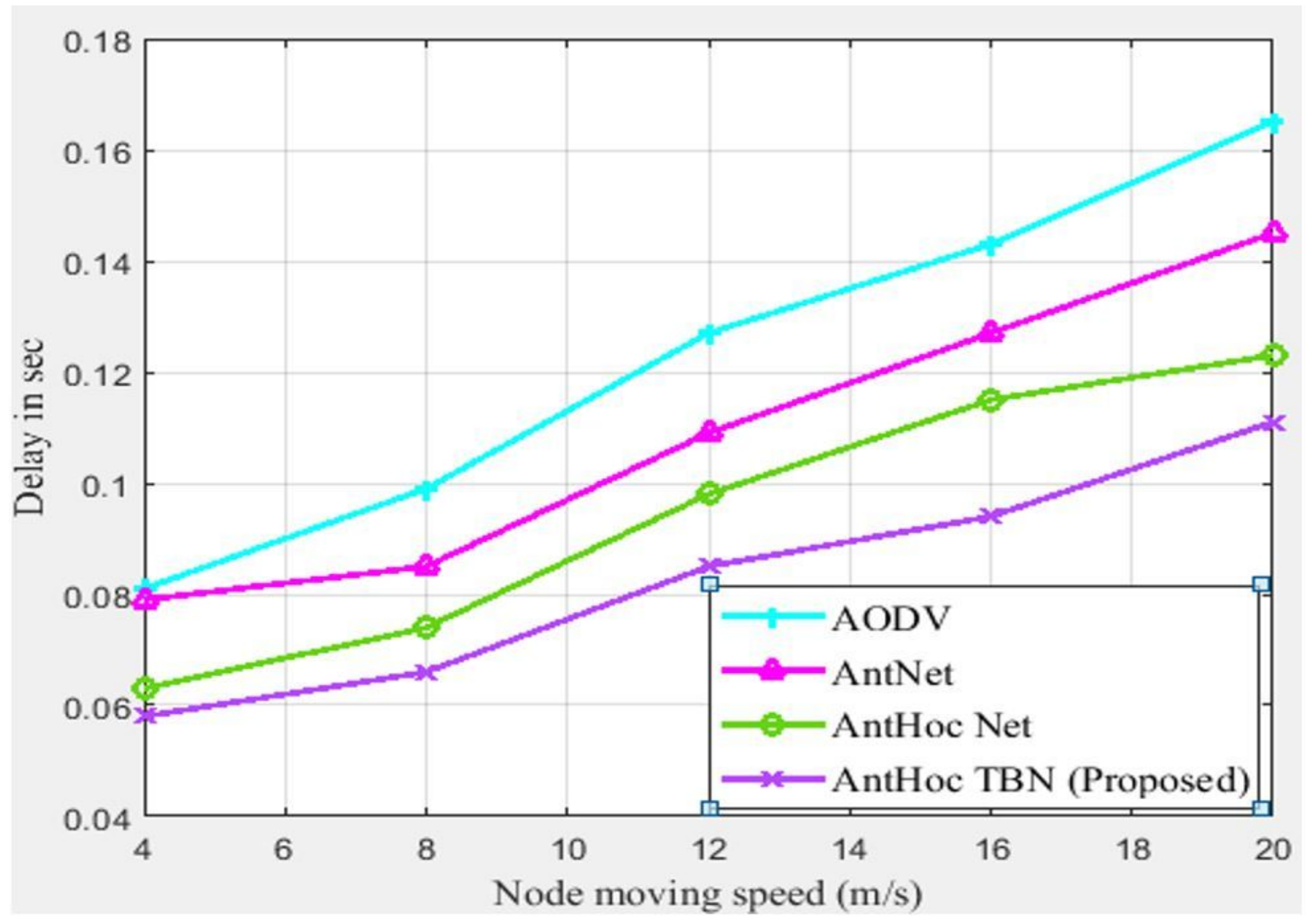

Figure 8

Delay versus node moving speed in $\mathrm{m} / \mathrm{s}$ 


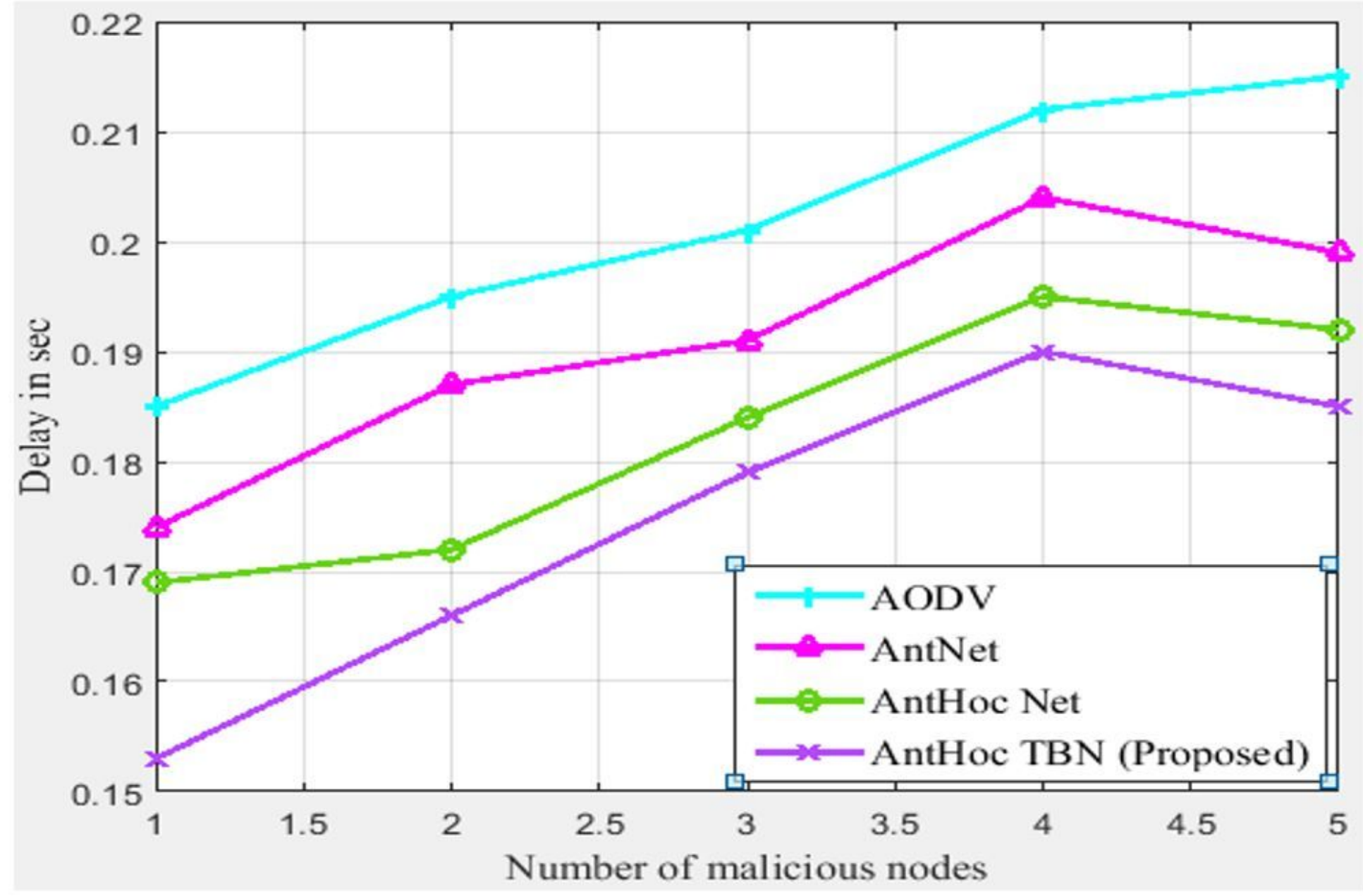

Figure 9

Delay versus number of malicious node 


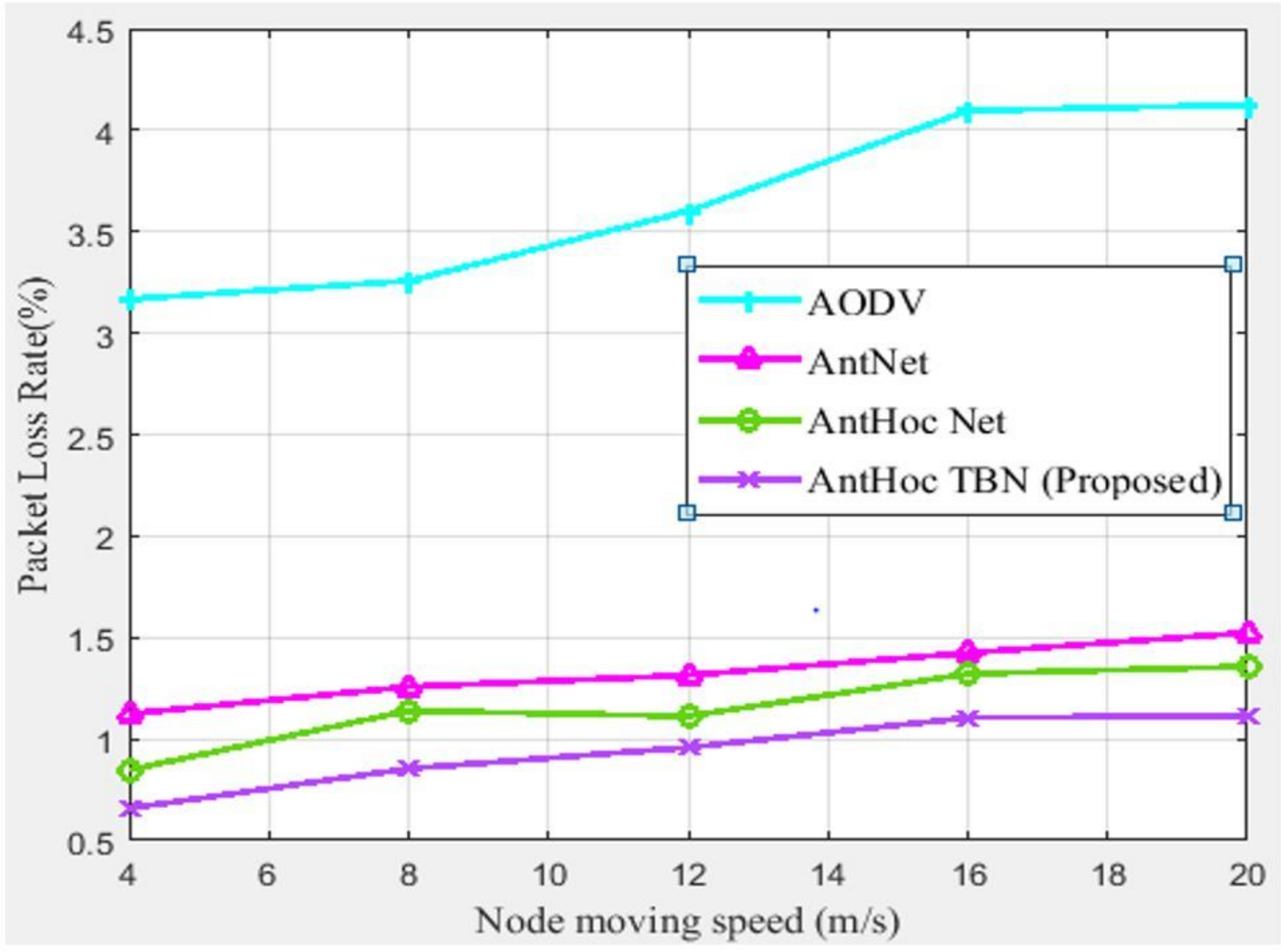

Figure 10

Packet loss versus node moving speed 


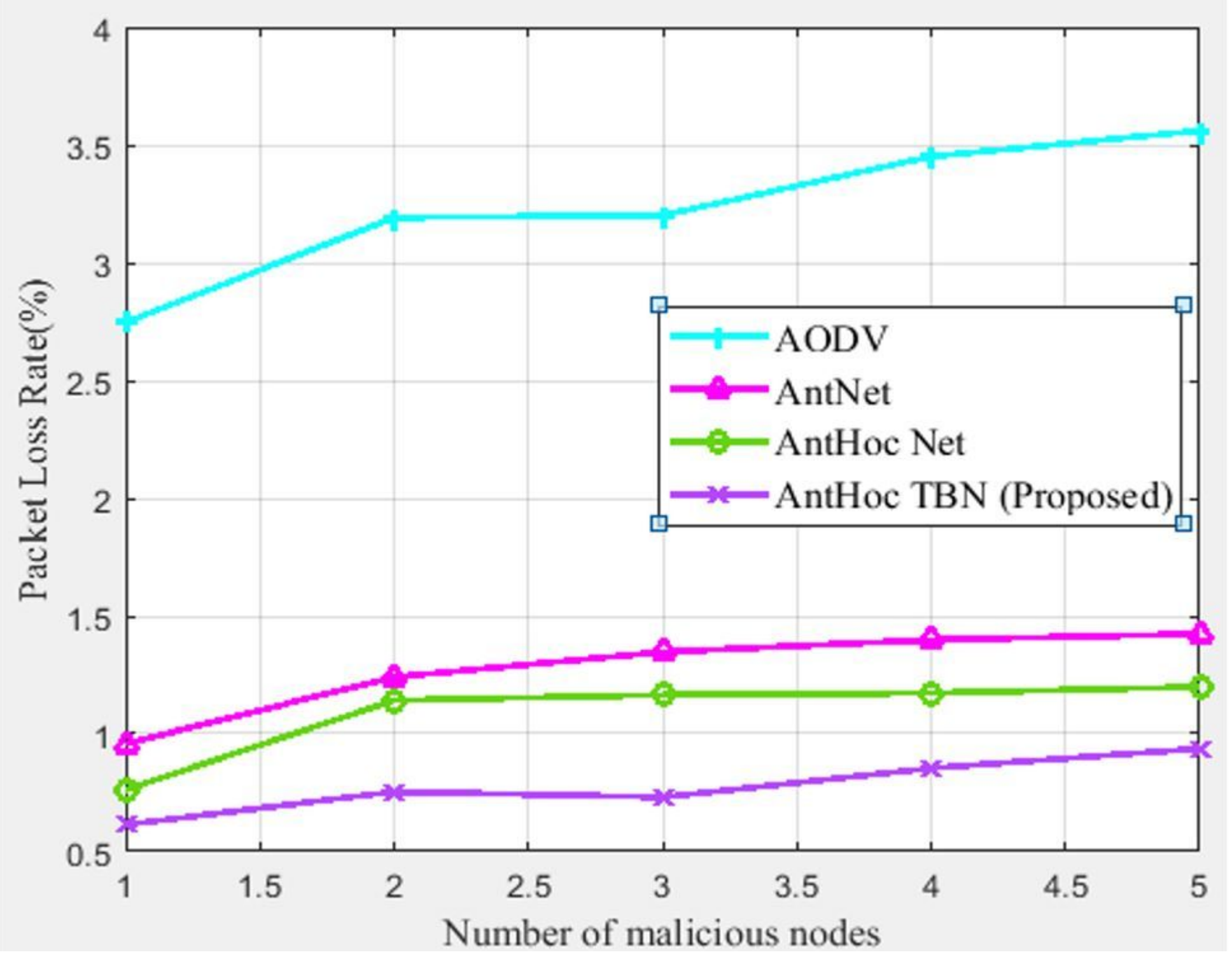

Figure 11

Packet loss versus number of malicious node 


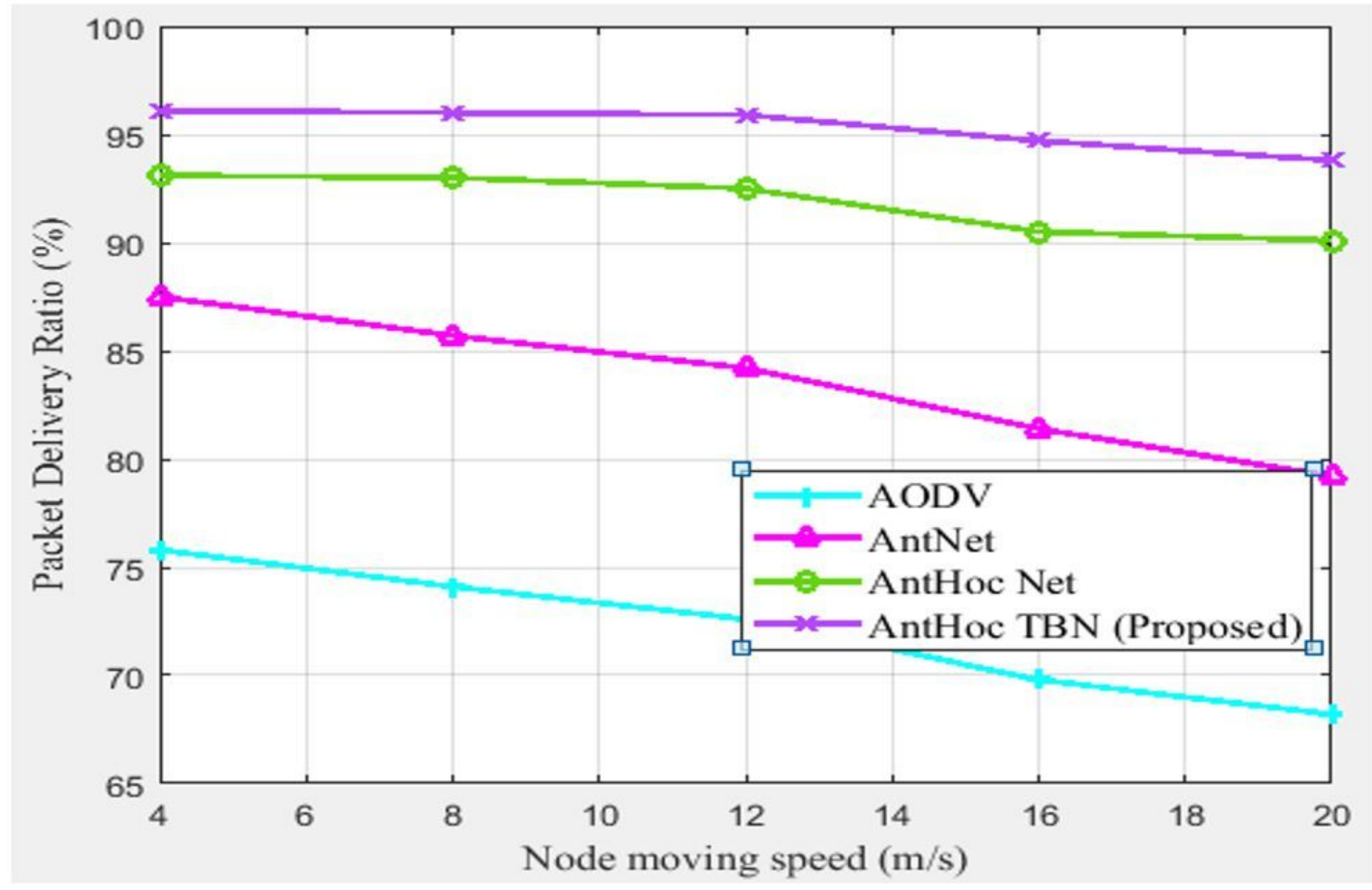

Figure 12

Packet delivery ratio versus node moving speed 


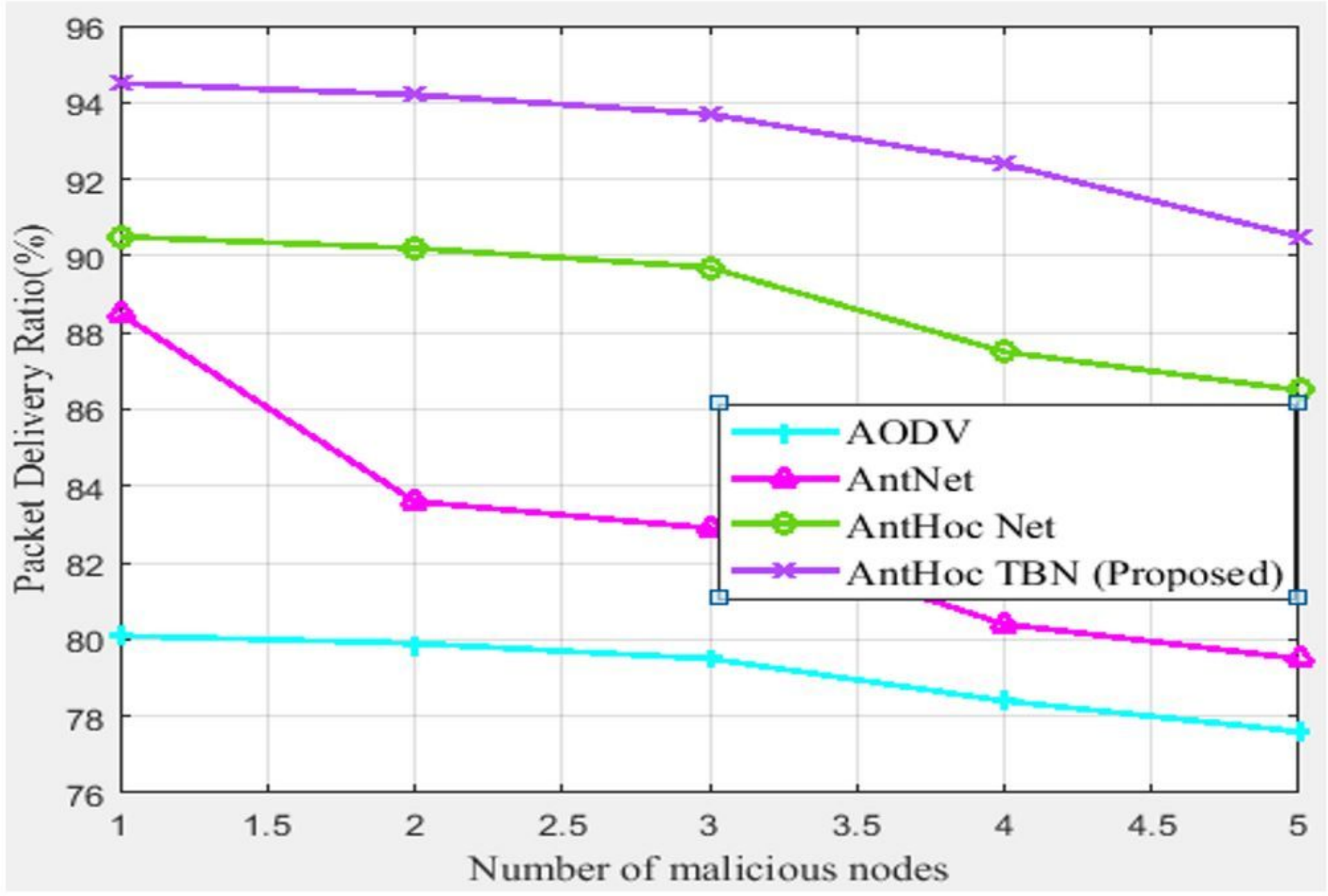

Figure 13

Packet delivery ratio versus malicious node 